\title{
Quantum black holes, localization, and the topological string
}

\author{
Atish Dabholkar, ${ }^{a, b}$ João Gomes ${ }^{a}$ and Sameer Murthy ${ }^{a, b}$ \\ ${ }^{a}$ Laboratoire de Physique Théorique et Hautes Energies (LPTHE), \\ Université Pierre et Marie Curie-Paris 6; CNRS UMR 7589, \\ Tour 24-25, 5ème étage, Boite 126, 4 Place Jussieu, 75252 Paris Cedex 05, France \\ ${ }^{b}$ Department of Theoretical Physics, Tata Institute of Fundamental Research, \\ Homi Bhabha Rd, Mumbai 400 005, India \\ E-mail: atish@lpthe.jussieu.fr, gomes@lpthe.jussieu.fr, \\ smurthy@lpthe.jussieu.fr
}

ABSTRACT: We use localization to evaluate the functional integral of string field theory on $A d S_{2} \times S^{2}$ background corresponding to the near horizon geometry of supersymmetric black holes in $4 \mathrm{~d}$ compactifications with $\mathcal{N}=2$ supersymmetry. In particular, for a theory containing $n_{v}+1$ vector multiplets, we show that the functional integral localizes exactly onto an ordinary integral over a finite-dimensional submanifold in the field space labeling a continuous family of instanton solutions in which auxiliary fields in the vector multiplets are excited with nontrivial dependence on $A d S_{2}$ coordinates. These localizing solutions are universal in that they follow from the off-shell supersymmetry transformations and do not depend on the choice of the action. They are parametrized by $n_{v}+1$ real parameters $\left\{C^{I} ; I=0, \ldots, n_{v}\right\}$ that correspond to the values of the auxiliary fields at the center of $A d S_{2}$. In the Type-IIA frame, assuming D-terms evaluate to zero on the solutions for reasons of supersymmetry, the classical part of the integrand equals the absolute square of the partition function of the topological string as conjectured by Ooguri, Strominger, and Vafa; however evaluated at the off-shell values of scalar fields at the center of $A d S_{2}$. In addition, there are contributions from one-loop determinants, brane-instantons, and nonperturbative orbifolds that are in principle computable. These results thus provide a concrete method to compute exact quantum entropy of these black holes including all perturbative and nonperturbative corrections and can be used to establish a precise relation between the quantum degeneracies of black holes and the topological string.

Keywords: Black Holes in String Theory, Superstrings and Heterotic Strings, AdS-CFT Correspondence

ARXIV EPRINT: 1012.0265 


\section{Contents}

1 Introduction and summary of results 1

2 Quantum entropy and $A d S_{2} / C F T_{1}$ correspondence $\quad 9$

2.1 Near horizon classical geometry $\quad 9$

$\begin{array}{lll}2.2 & \text { Functional integral for the quantum entropy } & 10\end{array}$

3 Superconformal symmetries and localization $\quad \mathbf{1 3}$

3.1 A review of localization of supersymmetric functional integrals 13

$\begin{array}{ll}3.2 & \text { Superconformal symmetries of the near horizon geometry } \\ \end{array}$

$\begin{array}{lll}4 & \text { Off-shell formulation of the theory } & 16\end{array}$

$\begin{array}{lll}4.1 & \text { Superconformal multiplets } & 17\end{array}$

$\begin{array}{llr}4.2 & \text { Superconformal action } & 19\end{array}$

5 Localization $\quad 21$

5.1 On-shell attractor geometry 21

$\begin{array}{lll}5.2 & \text { Localizing action and the localizing instantons } 22\end{array}$

$\begin{array}{lll}5.3 & \text { Renormalized action for the localizing instantons } & 26\end{array}$

$\begin{array}{ll}5.4 & \text { Evaluation of } \hat{W}(q, p) \\ \end{array}$

6 Quantum entropy and the topological string $\quad 29$

6.1 D-terms, hyper-multiplets, and evaluation of $W_{0}(q, p) \quad 30$

6.2 Non-perturbative corrections from orbifolds of $A d S_{2} \quad 31$

6.3 Relation to classical entropy function and the topological string 33

A Killing spinors in the attractor geometry 35

B Some aspects of the superconformal multiplet calculus $\quad 39$

C Boundary terms and supersymmetry of the renormalized action $\quad 41$

\section{Introduction and summary of results}

The entropy of a black hole remains one of the most precious and precise clues about the microscopic structure of quantum gravity. In a consistent quantum theory of gravity, the black hole entropy must have a statistical interpretation in terms of underlying microstates in the quantum Hilbert space of the theory. This is a very strong constraint on the underlying short distance degrees of freedom, since it must hold in any phase of the theory that admits black holes. For a theory under construction such as string theory, this requirement 
is a particularly useful guide especially since we do not yet know which phase or 'vacuum' of the theory may correspond to the real world. In such a situation, a profitable strategy is to focus on aspects of the theory that must be universally true in all phases of the theory. ${ }^{1}$

By now, there is a very good statistical understanding of the entropy of a large class of supersymmetric charged black holes in several compactifications of string theory, in the thermodynamic limit of large horizon area or large charges. In this limit, the leading Bekenstein-Hawking entropy [1,2] given by one quarter of the horizon area in Planck units precisely matches the logarithm of the degeneracy of the corresponding quantum microstates, as in the work of Strominger and Vafa [3]. Given this beautiful agreement to leading order, it is important to figure out what exact formula this is an approximation to, and how one might systematically compute corrections to the leading answer for finite charges or equivalently for finite horizon area. In the full quantum theory, both the macroscopic entropy and the microscopic degeneracy are expected to receive subleading corrections at the perturbative as well as nonperturbative level.

Finite size corrections to the leading Bekenstein-Hawking entropy are physically very interesting for the following reason. The Bekenstein-Hawking entropy is in a sense a bit too universal in that it is always given by a quarter of the horizon area. This is a consequence of the fact that it follows directly from the Einstein-Hilbert action which has a universal form in all theories of gravity at very long distances. Finite size corrections, on the other hand, arise from higher derivative corrections which are different in different phases. This dependence on the phase can yield useful information about different aspects of the short-distance theory. It is analogous to how one can learn about the fundamental microscopic Hamiltonian of a piece of metal by studying its thermodynamic properties in different phases which can vary depending on whether phonons or electrons make the dominant contribution.

With this motivation, our objective will be to develop the macroscopic computation of exact quantum entropy for supersymmetric black holes in a broad class of phases of string theory, namely vacua with $\mathcal{N}=2$ supersymmetry in four dimensions. In a theory with massless $n_{v}+1$ vector fields, a black hole is specified by a charge vector $\left(q_{I}, p^{I}\right)$ with $I=0, \ldots, n_{v}$. We would like to develop methods to systematically compute the quantum entropy for arbitrary finite values of the charges. For this purpose, we use the quantum entropy formalism developed by Sen $[4,5]$ which generalizes the notion of Wald entropy [6-8] in the case of extremal black holes using holography in the near horizon $A d S_{2}$ background. In this formalism, for a supersymmetric black hole of charge vector $(q, p)$, the macroscopic analog of the microscopic degeneracy $d(q, p)$ is given by the expectation value $W(q, p)$ of a Wilson line on the boundary of the near horizon $A d S_{2}$ with specific supersymmetric boundary conditions. Exact quantum entropy is thus given by a formal functional integral over all string fields on the $A d S_{2}$ background. We review this formalism in section 2.

Evaluating the formal expression for $W(q, p)$ by doing the string field theory functional integral is of course highly nontrivial. To proceed further we imagine first integrating out the infinite tower of massive string modes and massive Kaluza-Klein modes to obtain a

\footnotetext{
${ }^{1}$ The entropy itself will of course depend in an interesting way both on the phase and the states under consideration and is not expected to be universal. What is universal is the constraint that this entropy must have a statistical interpretation in terms of the independently computable quantum degeneracies.
} 
local Wilsonian effective action for the massless supergravity fields. To compute the exact quantum entropy, one has to then evaluate exactly this functional integral of a finite number of massless fields with $A d S_{2}$ boundary conditions using the full Wilsonian effective action keeping all higher derivative terms. This effective action can include in general not only perturbative corrections in $\alpha^{\prime}$ but also worldsheet instanton corrections. We can regard the ultraviolet finite string theory as providing a finite, supersymmetric, and consistent cutoff at the string scale. The functional integral with such a finite cut-off and a Wilsonian effective action containing all higher order terms is thus in principle free of ultraviolet divergences. This functional integral will be our starting point.

We are still left with the task of evaluating a complicated functional integral. The near horizon geometry preserves eight superconformal symmetries and moreover the action, measure, operator insertion, boundary conditions of the functional integral are all supersymmetric. This allows us to apply localization techniques [9] which simplifies the evaluation of the functional integral enormously. Localization requires identification of a fermionic symmetry of the theory that squares to a compact bosonic symmetry. Using this symmetry, one can then localize the functional integral onto the 'localizing submanifold' of bosonic field configurations invariant under the fermionic symmetry. We review the superconformal symmetries of the near horizon geometry and relevant aspects of localization in section 3.

Since localization is employed at the level of the functional integral and not just at the level of a classical action, it is important to use an off-shell formulation of supergravity. Off-shell formulations of supergravity are in general notoriously involved. At present a complete formulation of off-shell supergravity coupled to both vector and hyper multiplets is not known. To implement localization in a concrete manner, we therefore first consider in section 5 a simpler problem of computing this expectation value of the Wilson line in a truncated model of supergravity coupled only to vector multiplets with an action containing only F-terms which are chiral integrals over superspace. In particular we ignore possible D-terms and hyper multiplets, which are discussed later in section 6 . The action still contains an infinite number of higher derivative terms but all of F-type. We denote the corresponding functional integral for the expectation value of a Wilson line in this restricted theory on $A d S_{2}$ by $\hat{W}(q, p)$. Computation of $\hat{W}(q, p)$ is greatly simplified by the fact that, for vector multiplets in $\mathcal{N}=2$ supergravity, there exists an elegant off-shell formulation developed in [10-12], using the superconformal calculus. The spectrum consists of the Weyl multiplet that contains the graviton and the gravitini, $n_{v}+1$ vector multiplets, and one compensating multiplet that eliminates unwanted degrees of freedom. We review this formalism in section 4 .

The main result of the paper concerns the localization of the functional integral for $\hat{W}(q, p)$ which is derived in section 5. We now summarize the salient features of this computation and the answers that we have been able to obtain.

1. We choose a particular linear combination $Q$ of the superconformal superymmetries of the near horizon geometry and deform the functional integral by adding to the action a Q-exact term of the form $\lambda Q V$ for $V=(Q \Psi, \Psi)$ where $\Psi$ refer to all fermionic fields 
of the theory and $\lambda$ is a continuous parameter that we introduce. We discuss the localizing action $S^{Q}:=Q V$ in detail in section (5.2). As we will see, using off-shell supersymmetry variations is not only important for conceptual reasons but will turn out to be essential in this problem. In particular some auxiliary fields which are set to zero on the on-shell theory will play a critical role and develop a nontrivial position dependence for the localizing solutions.

2. By the usual arguments of localization reviewed in section 3.1, the functional integral localizes onto the critical points of the functional $S^{Q}$. We obtain a family of nontrivial instantons as exact solutions to the equations of motion that follow from extremization of $S^{Q}$. Since we use off-shell supersymmetry variations, these instanton solutions are completely universal and independent of the form of the physical action.

3. For these solutions, the scalar fields $X^{I}$ in the vector multiplets are no longer fixed at the attractor values $X_{*}^{I}$ but have a nontrivial position dependence in the interior of the $A d S_{2}$ given by

$$
X^{I}=X_{*}^{I}+\frac{C^{I}}{\cosh (\eta)}, \quad \bar{X}^{I}=\bar{X}_{*}^{I}+\frac{C^{I}}{\cosh (\eta)}
$$

in coordinates where the metric on $A d S_{2}$ takes form $d s^{2}=\sinh ^{2}(\eta) d \theta^{2}+d \eta^{2}$. Auxiliary fields in the vector multiplets are excited in such a way that the $\mathrm{Q}$ supersymmetry is preserved. The family of solutions is parametrized by real parameters $\left\{C^{I}\right\}, I=1, \ldots, n_{v}+1$, which correspond to the values of the auxiliary fields in the vector multiplets at the center of $A d S_{2}$. The infinite-dimensional functional integral thus localizes onto a finite number of ordinary bosonic integrals over the $\left\{C^{I}\right\}$.

4. Many D-terms are expected to evaluate to zero on these solutions because of the nonrenormalization theorem of [13]. We assume this to be true more generally and restrict our attention to F-type action. ${ }^{2}$ Such actions are completely specified by specifying a single holomorphic prepotential $F\left(X^{I}, A\right)$ where $X^{I}$ are scalars in the vector multiplet and $A$ is the auxiliary field in the Weyl multiplet. For such actions, we evaluate the renormalized action $S_{\text {ren }}$ for the Q-invariant localizing instanton configurations exactly as a function of the $\left\{C^{I}\right\}$ following the prescription in $[4,5]$. This action takes the form

$$
\mathcal{S}_{\text {ren }}(\phi, q, p)=-\pi q_{I} \phi^{I}+\mathcal{F}(\phi, p)
$$

with $\mathcal{F}$ given by

$$
\mathcal{F}(\phi, p)=-2 \pi i\left[F\left(\frac{\phi^{I}+i p^{I}}{2}\right)-\bar{F}\left(\frac{\phi^{I}-i p^{I}}{2}\right)\right] .
$$

Note that $S_{\text {ren }}(\phi, q, p)$ equals precisely the classical entropy function $\mathcal{E}(e, q, p)[4,5]$ but with electric fields replaced by the linear combination $\phi^{I}:=e_{*}^{I}+2 C^{I}$ where $e_{*}^{I}$ are the attractor values of the electric field.

\footnotetext{
${ }^{2}$ If this assumption is not true then the contribution of the D-terms can be systematically taken into account by simply evaluating these terms on our localizing solution.
} 
5. We would like to emphasize that even though $S_{\text {ren }}$ and $\mathcal{E}$ have the same functional form, their physical origin and meaning is very different. The entropy function is essentially a classical and on-shell object. Only its extremum which determines the classical attractor values and its value at the extremum which determines the Wald entropy have physical meaning. By contrast, $S_{\text {ren }}$ is an intrinsically off-shell object valid for values of the fields far away from the classical attractor values. Now, as long as $S_{\text {ren }}$ has the same extremum and the value at the extremum as $\mathcal{E}$ but differs from $\mathcal{E}$, it would reproduce the semi-classical results. Hence, a priori, away from the extremum the two functions could have been very different. It is thus something of a surprise that the nontrivial computation in section 5 for the off-shell instantons yields the same answer as the classical entropy function.

6. The infinite dimensional functional integral thus reduces to the following finite dimensional integral

$$
\hat{W}(q, p)=\int_{\mathcal{M}_{Q}} e^{-\pi \phi^{I} q_{I}} e^{\mathcal{F}(\phi, p)}\left|Z_{\text {inst }}\right|^{2} Z_{\operatorname{det}}[d C]_{\mu}
$$

The measure of integration over $[d C]_{\mu}$ is computable from the original measure $\mu$ of the functional integral of massless fields of string theory by standard collective coordinate methods. The factor $Z_{\text {det }}$ are the one-loop determinants of the quadratic fluctuation operator around the localizing instanton solution. Such one-loop determinant factors in closely related problems have been computed in [14, 15]. This computations are straightforward in principle but technically involved and we defer their detailed discussion to a forthcoming publication [16].

7. We have included a term $\left|Z_{\text {inst }}\right|^{2}$ to indicate the contribution of brane-instantons which in general will be present in the full string theory computation. Supersymmetric configurations in $A d S_{2} \times S^{2}$ typically correspond to instantons localized at the north pole of $S^{2}$ with counting function $Z_{\text {inst }}$ and anti instantons localized at the south pole with counting function $\bar{Z}_{\text {inst }}[14,17]$. Note that nonperturbative instantons in one duality frame may be incorporated as worldsheet instantons in another duality frame, and hence the separation between the classical piece and instanton piece is frame-dependent. We keep it general at this stage to underscore the fact that the physics of black hole horizons is also frame-dependent. This is a consequence of the fact that for the same asymptotic states, some degrees of freedom that are horizon degrees of freedom in one frame may be external to the horizon in another frame $[18,19]$.

Independent of our original motivation of computing quantum entropy of black holes, results in section 5 could be viewed simply as results about the localization of this functional integral of supergravity coupled to vector multiplets keeping only F-terms. Equation (1.4) represents a remarkable reduction of a complicated functional integral of gravity onto an ordinary integral with an integrand that depends on the prepotential in a particularly simple way. 
In section 6 , we proceed to the evaluation of $W(q, p)$ using these results for $\hat{W}(q, p)$. For this purpose it is necessary to make the assumption that the hyper multiplets and D-terms do not contribute. We examine this in some detail in section 6.1 in view of the nonrenormalization theorem of [13]. We then discuss in section 6.2 the subleading gravitational saddle points which will in general give nonperturbative contributions in addition to those from brane-instantons. We discuss how these saddle point can be taken into account in a general setup. We conclude section 6.3 with comments about the connection with the topological string and the Donaldson-Thomas invariants. Before summarizing the general structure of the localization integral, we would like to clarify two common misconcopetions.

- Choice of the ensemble: The large area limit is the thermodynamic limit. The leading answer for the entropy in this limit is the Bekenstein- Hawking entropy which does not depend on the choice of the ensemble. However, the subleading corrections, which are the finite size effects, depend sensitively on the ensemble. It is thus necessary to determine which ensemble is natural on the macroscopic side. The natural ensemble from the perspective of the $A d S_{2}$ boundary conditions $[4,5]$ is the microcanonical one. ${ }^{3}$ This follows from the fact that in $A d S_{2}$, the Coulomb potential grows linearly at infinity. As a result, in $A d S_{2}$, the fluctuation of charge corresponds to a nonnormalizable mode unlike say in $A d S_{5}$, and must be held fixed at the boundary.

- Index vs degeneracy: The entropy of a black hole is a thermodynamic quantity and accoding to Boltzmann relation it should be compared with the absolute number of states. The supersymmetric techniques that we will be using would seem more appropriate for the computation of an index and not the entropy or the absolute number. However, for a black hole with an $A d S_{2}$ near horizon geometry that preserves at least four supersymmetries, the index equals the absolute number. This follows from the following reasoning [22]. Demanding closure of the algebra containing the $\mathrm{SU}(1,1)$ isometries of the $A d S_{2}$ together with the four supersymmetries implies that the symmetry is a supergroup $\mathrm{SU}(1,1 \mid 2)$ which contains an $\mathrm{SU}(2)$ symmetry. This implies that the black hole horizon has rotational symmetry. ${ }^{4}$ In a thermodynamic ensemble, this could either mean that its spin $J$ is zero or the chemical potential $\mu$ conjugate to the spin is zero. However, the boundary conditions for the choice of the ensemble discussed above require that we hold $J$ fixed which appears as a charge in $A d S_{2}$ and not the chemical potential. This implies that for the microstates associated

\footnotetext{
${ }^{3}$ In some cases it is possible that both boundary conditions are physically allowed as in the BreitenlohnerFreedman window in higher dimensions. The two choices of the boundary conditions lead to physically distinct theories whose partition functions could be regarded to be related to each other by a Legendre transform [20]. Thus it may be possible to use the mixed ensemble in special cases, especially to compare with the OSV conjecture [21] where the mixed ensemble arises naturally. However, in generic examples the sum over charges may not be convergent and it is not clear if the mixed ensemble can be defined beyond an asymptotic expansion. For a detailed discussion of the comparison of the two ensembles see [5]. In this paper we use the microcanonical boundary conditions which are most general and natural in the $A d S_{2}$ set-up.

${ }^{4}$ The well-known fact that there are no spinning supersymmetric black holes in four dimensions is a consequence of this fact.
} 
with the horizon of a single black hole

$$
\operatorname{Tr}(-1)^{F}:=\operatorname{Tr}\left(e^{2 \pi i J}\right)=\operatorname{Tr}(1),
$$

and hence the index equals the absolute number. Since our computation will be near the horizon of a single black hole, the argument above shows that it is justified to use supersymmetric localization methods even for the computation of the absolute number. If one wishes to compare this with a microscopic computation which is often done for an index at asymptotic infinity it is necessary to define an analogous index on the macroscopic side using our results here as an input. For a more detailed discussion see [23].

Putting these various ingredients together, the final answer for the quantum entropy is of the general form

$$
W(q, p)=\sum_{s} W_{s}(q, p),
$$

where $s$ is a non-negative integer labeling various orbifolds with $s=0$ being the unorbifolded theory. The term $W_{0}$ denotes the functional integral on unorbifolded $A d S_{2}$ which gives the leading contribution and will be discussed in section 6.1. In the semilclassical limit, $W_{0}$ will scale as $\exp \left(S_{\text {Wald }}\right)$ whereas $W_{s}$ for nonzero $s$ will scale as $\exp \left(S_{\text {Wald }} / c\right)$ where $c$ is the order of the orbifold. These terms are thus exponentially subleading compared to $W_{0}$ and will be discussed in section 6.2. We would like to emphasize two features of this answer.

1. If D-terms can be ignored for reasons of supersymmetry, then we expect $W_{0}$ will equal $\hat{W}$ given by (1.4). In the Type-IIA frame, the world sheet instanton corrections are incorporated in the prepotential computed by the topological string. Moreover, it is likely that there are no spacetime instanton contributions from wrapped D-brane or NS5-branes because their action would have to depend on the dilaton which is in the hyper-multiplet. In other words, such instantons wrapping an internal cycle of the Calabi-Yau but localized at the poles of $S^{2}$ may not contribute to the supersymmetric functional integral. We have not analyzed this in detail but if true, it would imply that

$$
W_{0}(q, p)=\int_{\mathcal{M}_{Q}} e^{-\pi \phi^{I} q_{I}}\left|Z_{\mathrm{top}}(\phi, p)\right|^{2} Z_{\operatorname{det}}[d C]_{\mu} .
$$

The term $\left|Z_{\text {top }}\right|^{2}$ is precisely of the form envisaged by Ooguri-Strominger-Vafa [21]! It should be emphasized though that in this derivation, the topological string partition function in (1.7) is evaluated for the values of the scalar fields at the center of $A d S_{2}$ and not at the boundary of $A d S_{2}$. Since the scalar fields are moving away from the attractor values, they are no longer at the minimum of the entropy function and hence are thus 'climbing up' the entropy function away from the critical point.

2. As mentioned above, for a consistent treatment of the $A d S_{2}$ path integral it is necessary to work in the microcanonical ensemble because in $A d S_{2}$ Coulomb potential is a a nonnormalizable mode. Various fields approach their attractor values at the 
boundary in this fixed charged sector. In particular, the electric fields at the boundary do not fluctuate and remain fixed at their attractor values $e_{*}$. For our localizing instanton solutions the fields move off-shell away from the attractor values inside $A d S_{2}$ and it is the value of these fields at the center of $A d S_{2}$ that is allowed to fluctuate and can be integrated over as in (1.7). This derivation thus requires off-shell supergravity in an essential way.

3. It has been pointed out that agreement with statistical entropy requires modifying the OSV conjecture by including additional measure factors and nonperturbative corrections [24-29]. Our localizing solution provides a well-defined starting point to define these corrections systematically on the macroscopic side as we discuss below. See section 6.3 for a more detailed discussion of the relation to the topological string and comparison with earlier work.

4. Note that the range of integration is determined by the localization analysis and goes from $-\infty$ to $+\infty$ for all $C$. Since the measure factor and $Z_{\text {det }}$ follow algorithmically from the original measure of the string theory spacetime fields, the resulting answer is guaranteed to respect all symmetries. In particular, even though the renormalized action is Wilsonian and given in terms of holomorphic prepotential, one expects from the $A d S_{2} / C F T_{1}$ correspondence that holomorphic anomalies [30-32] are taken into account systematically by the integral (1.7) so that the resulting degeneracies respect all duality symmetries.

5. The higher orbifold contributions in (1.6) appear to play an important role if the macroscopic analysis is to reproduce the known microscopic answers in the $\mathcal{N}=4$ examples [16]. In particular, their contribution would be essential to ensure that the resulting sum is an integer. From this point of view as well, it is more natural to work in the microcanonical ensemble than invert (1.7) and work in a mixed ensemble.

To summarize, in this paper we have solved one major piece of the puzzle in the evaluation of the black hole functional integral. We have determined which off-shell field configurations to integrate over consistent with the $A d S_{2}$ boundary conditions. We have obtained explicit analytic expressions for the localizing instanton solutions and the renormalized action evaluated on these instantons. These solutions thus give a well-defined starting point to evaluate various corrections to the semi-classical results. Further work is necessary to determine one-loop contributions and the measure [16]. This computation is technically involved but we would like to emphasize that given the localizing action and the localizing instantons this step is essentially algorithmic. Computation of braneinstantons in general case will be more complicated since it will depend on the details of the compactification but can simplify in specific examples and in specific duality frames.

Finally, we note that in models where exact microscopic degeneracies $d(q, p)$ are known from independent computations, our macroscopic computation can be tested against the microscopic answer. Such a comparison for half-BPS small black holes and quarter-BPS big black holes in $\mathcal{N}=4$ compactifications will be reported in a forthcoming publication [16]. 
In these examples, all the ingredients of the macroscopic computation discussed in this paper play an important role. With $\mathcal{N}=4$ supersymmetry, all three terms in the integrand $\exp (\mathcal{F}), Z_{\text {det }}$ and $Z_{\text {inst }}$ can be computed almost completely. Moerover, the resulting integral representation can be immediately be put a form that that agrees in remarkable detail with the microscopic degeneracies including all perturbative and nonperturbative corrections. These results can thus be seen as a partial post-facto justification for some of the assumptions made in section 6 .

\section{Quantum entropy and $A d S_{2} / C F T_{1}$ correspondence}

The quantum entropy formalism [4, 5] generalizes the Wald entropy formula to include quantum corrections to black hole entropy in a consistent quantum theory of gravity such as string theory. It is formulated in general for any extremal black holes whose near horizon geometry has an $A d S_{2}$ factor. We would now like to apply this formalism to four-dimensional supersymmetric black hole whose near-horizon geometry is of the form $A d S_{2} \times S^{2} \times K$ where $K$ is a three complex dimensional Calabi-Yau manifold of string compactification.

Since the essential physics of the quantum entropy concerns the $A d S_{2}$ factor, for the rest of this section we will dimensionally reduce all the way to two dimensions onto $A d S_{2}$. One can regard the full theory as a two-dimensional theory of gravity interacting with an infinite set of fields keeping all massive modes. The massless sector consists of the 2D metric, a set of gauge fields $A^{i}$ with field strengths $F^{i}$, and matter fields $\phi^{a}$ which include the moduli of $K$, as well as the fluxes through the various cycles in the 'internal' geometry $S^{2} \times K$. The electric charge of the four-dimensional black hole is represented by the gauge fields, and the magnetic charges which correspond to fluxes through the $S^{2}$ are represented as fixed parameters of the theory living on the $A d S_{2}$ geometry.

\subsection{Near horizon classical geometry}

The most general near horizon configuration for the massless fields consistent with the $\mathrm{SL}(2, \mathbb{R})$ isometry of $A d S_{2}$ is:

$$
d s^{2}=v\left[-\left(r^{2}-1\right) d t^{2}+\frac{d r^{2}}{r^{2}-1}\right], \quad F^{i}=e^{i} d r \wedge d t, \quad \phi^{a}(t, r)=\phi_{0}^{a},
$$

where $v, e^{i}$ and $\phi_{0}^{a}$ are constants. This is the metric of an $A d S_{2}$ black hole [33-36] with horizon at $r=1$. It is locally isometric to $A d S_{2}$ and the region $r>1$ covers a triangular wedge extending halfway from the boundary into global $A d S_{2}$ [36]. An analytic continuation $t=-i \theta$ leads to the Euclidean metric

$$
d s^{2}=v\left[\left(r^{2}-1\right) d \theta^{2}+\frac{d r^{2}}{r^{2}-1}\right], \quad F^{i}=-i e^{i} d r \wedge d \theta, \quad \phi^{a}(\theta, r)=\phi_{0}^{a} .
$$

This metric is non-singular at the erstwhile horizon $r=1$ provided the Euclidean time coordinate $\theta$ is periodic modulo $2 \pi$. In the gauge $A_{r}^{i}=0$, the gauge fields are given by

$$
A^{i}=-i e^{i}(r-1) d \theta,
$$


where the constant term ensures that the Wilson line $\oint_{S^{1}} A^{i}$ around the thermal circle vanishes at the horizon $r=1$. This is needed for regularity since the thermal circle contracts to zero size.

It is worth emphasizing that it is important to use the form of the metric in (2.1) with two separate horizons at $r= \pm 1$ which corresponds to the Jackiw-Teitelboim black hole [33-35]. Physically this corresponds to staying close to the black hole horizon as one takes the near horizon limit in which the $A d S_{2}$ throat becomes infinitely long. Upon Euclidean continuation this covers the entire upper half plane or the Poincaré disk which has the topology of a disk and hence Euler character one. In the Gibbons-Hawking formalism, the entropy of the black hole is proportional to the Euler character of the near horizon in the $(r, t)$ plane and hence one obtains finite entropy. If we use instead the metric

$$
d s^{2}=v\left[\rho^{2} d \theta^{2}+\frac{d \rho^{2}}{\rho^{2}}\right]
$$

with periodic $\theta$ then this covers only a strip in the upper half plane with two edges identified. The geometry then has a topology of a cylinder, or a punctured disk, which has Euler character zero, and hence vanishing entropy [37]. For applications in string theory it has been clear that an extremal black hole should really be thought of as a limit of a nonextremal black hole in which case it has zero temperature but nonzero entropy. This corresponds to choosing the metric as in (2.1).

\subsection{Functional integral for the quantum entropy}

The quantum entropy is defined by a functional integral over all field configurations which asymptote to the $A d S_{2}$ Euclidean black hole (2.2) with the fall-off conditions [38]

$$
\begin{aligned}
d s_{0}^{2} & =v\left[\left(r^{2}+\mathcal{O}(1)\right) d \theta^{2}+\frac{d r^{2}}{r^{2}+\mathcal{O}(1)}\right], \\
\phi^{a} & =u^{a}+\mathcal{O}(1 / r), \quad A^{i}=-i e^{i}(r-\mathcal{O}(1)) d \theta,
\end{aligned}
$$

which are invariant under an action of the Virasoro algebra. In particular, in contrast to higher dimensional instances of the AdS/CFT correspondence, the mode of the gauge field corresponding to the electric field grows linearly (or is 'non-normalizable') and must be kept fixed, while the mode corresponding to the electric potential is constant (or is 'normalizable'), and allowed to fluctuate. Since the asymptotic value of the electric field is determined by the charge of the black hole by Gauss law

$$
q_{i}=\frac{\partial(v \mathcal{L})}{\partial e^{i}}
$$

this is equivalent to holding the charge fixed. The asymptotic values of the parameters of the metric and the scalars are determined purely in terms of the charges by the attractor mechanism. The constants $v, e^{i}, u^{a}$ which set the boundary conditions of the path integral must therefore be set to their attractor values $v_{*}, e_{*}^{i}, u_{*}^{a}$ respectively. The quantum entropy is thus purely a function of the electric charges $q_{i}$. This was defined by [5] as the functional 
integral with an insertion of the Wilson line:

$$
W(q, p)=\left\langle\exp \left[-i q_{i} \int_{0}^{2 \pi} A_{\theta}^{i} d \theta\right]\right\rangle_{\mathrm{AdS}_{2}}^{\text {finite }} .
$$

Note that in the classical limit, this constant mode of the gauge field gets determined in terms of the attractor electric field $e_{*}^{i}$ by the smoothness condition on the classical gauge field but in the quantum theory it is free to fluctuate.

We will now explain the meaning of the superscript finite in the above functional integral. The action entering into this functional integral is of the form

$$
S_{\text {bulk }}+S_{\text {bdry }}
$$

where the actions ${ }^{5}$

$$
S_{\text {bulk }}=\int \mathcal{L}_{\text {bulk }} \sqrt{g} d r d \theta, \quad S_{\text {bdry }}=\int \mathcal{L}_{\text {bdry }} \sqrt{g_{\text {ind }}} d \theta
$$

are expressed in terms of local Lagrangian densities, the measure in the boundary term coming from the induced metric on the boundary. The integral for the bulk action over $r$ suffers from an obvious infrared divergence due to the infinite volume of the $A d S_{2}$. The superscript finite in (2.7) refers to the following prescription for regulating and renormalizing this divergence.

First, one enforces a cutoff at a large $r=r_{0}$. This cutoff which respects the angular symmetry seems to be special, but the conclusions below have been shown to be independent of the details of the cutoff [22]. The bulk Lagrangian density $\mathcal{L}_{\text {bulk }}$ is the full local classical Lagrangian of the theory including all massive fields. Since $\mathcal{L}_{\text {bulk }}$ is a local functional of the fields, the bulk effective action evaluated on a certain field configuration has the form

$$
S_{\mathrm{bulk}}=C_{0} r_{0}+C_{1}+\mathcal{O}\left(r_{0}^{-1}\right),
$$

with $C_{0}, C_{1}$ independent of $r_{0}$.

The boundary action is the boundary Lagrangian $\mathcal{L}_{\text {bdry }}$ multiplied by the the proper length $L \sim 2 \pi \sqrt{v} r_{0}$ of the boundary which goes to infinity as $r_{0} \rightarrow \infty$. $\mathcal{L}_{\text {bdry }}$ is a local gauge invariant functional of the fields of the theory. Using the asymptotic form of the fields (2.5), one obtains that the boundary action has a form like (2.10) with coefficients that only depend on the classical values of the various fields in the problem which are held fixed.

One now chooses the boundary counterterms such that the piece linear in $r_{0}$ in the integrand vanishes, as is standard in the procedure of holographic renormalization. In particular, one can subtract the constant piece $C_{0} r_{0}$ from the action simply by using an appropriate boundary cosmological constant as in [5]. This ensures that the boundary Hamiltonian of the $C F T_{1}$ dual to $A d S_{2}$ is zero. From the above argument, this boundary

\footnotetext{
${ }^{5}$ The signs in the first equation for the bulk action is chosen in accord with the Euclidean continuation from the Minkowski theory [4], the sign in the second equation is a convention since it is a one dimensional Euclidean problem, which we have fixed.
} 
cosmological constant is a function of the classical values of the fields. There can be of course other finite parts of the boundary action which depend on the fluctuating parts of the fields, this will be part of the full definition of the quantum entropy function. We shall comment on them below.

It is convenient to incorporate also the Wilson line into the renormalized action and include counterterms to cancel the divergences in the Wilson line. One can then take the limit $r_{0} \rightarrow \infty$, and define the finite part of the path integral unambiguously as $e^{-S_{\text {ren }}}$. We refer to this finite piece $S_{\text {ren }}$ as the renormalized action, which in general is a functional of all the fields. We thus have the definition

$$
\mathcal{S}_{\text {ren }}:=\mathcal{S}_{\text {bulk }}+\mathcal{S}_{\text {bdry }}-i q_{i} \int_{0}^{2 \pi} A_{\theta}^{i} d \theta
$$

In the classical limit, the functional integral (2.7) is dominated by the saddle point where all fields take their classical values (2.2). In this case, the path integral reduces to

$$
\left\langle\exp \left[-i q_{i} \int_{0}^{2 \pi} A^{i} d \theta\right]\right\rangle=\exp \left(S_{\mathrm{bulk}}+S_{\mathrm{bdry}}-i q_{i} \int_{0}^{2 \pi} A_{\theta}^{i} d \theta\right)
$$

where $S_{\text {bulk }}$ and $S_{\text {bdry }}$ are as above. In this case, one can simply evaluate the bulk Lagrangian at the constant classical values (2.1) to get

$$
\begin{aligned}
S_{\text {bulk }} & =\int_{0}^{2 \pi} d \theta \int_{1}^{r_{0}} d r v \mathcal{L}=2 \pi\left(r_{0}-1\right) v \mathcal{L}, \\
S_{\text {bdry }} & =-2 \pi r_{0}\left(v \mathcal{L}-q_{i} e^{i}\right)+\mathcal{O}\left(1 / r_{0}\right), \quad-i q_{i} \int_{0}^{2 \pi} A_{\theta}^{i} d \theta=-2 \pi q_{i} e^{i}\left(r_{0}-1\right) .
\end{aligned}
$$

After the above regulation and renormalization procedure, one has

$$
W(q, p) \sim \exp \left[2 \pi\left(q_{i} e^{i}-v \mathcal{L}\right)\right] \equiv \exp \left[S_{\mathrm{Wald}}(q, p)\right]
$$

where it is understood that the middle term is evaluated at the attractor values of the fields. Since the attractor values of various fields and in particular the electric fields are determined by extermization of the classical action, one can define the entropy function

$$
\mathcal{E}(e, p, q):=2 \pi\left(q_{i} e^{i}-v \mathcal{L}(e, p)\right) .
$$

Here we have fixed the scalars to their attractor values but kept the electric fields as variables. By virtue of its construction, the classical attractor values $e_{*}(q, p)$ of the electric fields can be found at the extremum of $\mathcal{E}$ which are determined entirely in terms of the charges. As shown in [4], the value of the entropy function at the extremum equals the Bekenstein-Hawking-Wald entropy of the extremal black hole.

In the case of supersymmetric black holes, we should use a supersymmetric version of this Wilson line which requires the addition of another boundary term to the Wilson line. We find that the bulk Lagrangian is also supersymmetric only up to a boundary term which has to be cancelled by adding a boundary counterterm. Both these additional boundary terms are field dependent. However, it turns out that the sum of the two additional 
boundary terms is equal to a constant diverging linearly with $r_{0}$, and moreover, it is precisely the constant required to cancel the infrared divergence of the original bulk action plus Wilson line. As a result we can express the total functional integral in a manifestly supersymmetric manner, at the same time using the naive operational definition above. We will discuss these issues and the supersymmetry of the functional integral in more detail in section 5.3 and section C.

In the full quantum theory, there will be corrections to the classical answer which are of two kinds. The first type of correction will arise from evaluating the functional integral around the $A d S_{2}$ geometry. One can try to evaluate it in a saddle point approximation, but this can at best give an asymptotic expansion and one can never access large fluctuations in field space. We shall overcome this using the technique of localization in the context of supersymmetric theories. This will allow us to evaluate the functional integral in the $A d S_{2}$ background exactly. We discuss this in the next sections.

The second type of correction comes from subleading orbifold saddle points that play an important role. Keeping these subleading saddle points which are much smaller than the power law corrections to the leading saddle point cannot be justified in an asymptotic expansion, but the exact evaluation of the functional integral allows us here to consistently deal with subleading saddles. The full functional integral localizes onto a discrete series, and for each term in the series we obtain an exact finite dimensional integral which accesses large fluctuations in field space. We discuss the general form of the localizing integral in section 6.2.

\section{Superconformal symmetries and localization}

We start with a brief review in section 3.1 of the localization techniques [39-44] to evaluate supersymmetric functional integrals. In section 3.2 we review the superconformal symmetries of the attractor geometry and how localization can be applied in the present context.

\subsection{A review of localization of supersymmetric functional integrals}

Consider a supermanifold $\mathcal{M}$ with an integration measure $d \mu$. Let $Q$ be an odd (fermionic) vector field on this manifold that satisfies the following two requirements:

- $Q^{2}=H$ for some compact bosonic vector field $H$,

- The measure is invariant under $Q$, in other words $\operatorname{div}_{\mu} Q=0$.

The divergence of the fermionic vector field is the natural generalization of ordinary divergence, which satisfies in particular ${ }^{6}$

$$
\int_{\mathcal{M}} d \mu Q(f)=-\int_{\mathcal{M}} d \mu\left(\operatorname{div}_{\mu} Q\right) f
$$

\footnotetext{
${ }^{6}$ For a bosonic vector field $V$ and for a measure determined by a metric $g$, this corresponds to the identity $\int d x \sqrt{g} V^{m} \partial_{m} f=-\int d x \partial_{m}\left(\sqrt{g} V^{m}\right) f=-\int d x \sqrt{g}\left(\nabla_{m} V^{m}\right) f$ when the boundary contributions vanish.
} 
for any function $f$. Hence, the second property implies $\int_{\mathcal{M}} d \mu Q(f)=0$ for any $f$. We would like to evaluate an integral of some $Q$-invariant function $h$ and a Q-invariant action $S$

$$
I:=\int_{\mathcal{M}} d \mu h e^{-\mathcal{S}}
$$

To evaluate this integral using localization, one first deforms the integral to

$$
I(\lambda)=\int_{\mathcal{M}} d \mu h e^{-\mathcal{S}-\lambda Q V}
$$

where $V$ is a fermionic, H-invariant function which means $Q^{2} V=0$ and $Q V$ is Q-exact. One has

$$
\frac{d}{d \lambda} \int_{\mathcal{M}} d \mu h e^{-\mathcal{S}-\lambda Q V}=\int_{\mathcal{M}} d \mu h Q V e^{-\mathcal{S}-\lambda Q V}=\int_{\mathcal{M}} d \mu Q\left(h e^{-\mathcal{S}-\lambda Q V}\right)=0,
$$

and hence $I(\lambda)$ is independent of $\lambda$. This implies that one can perform the integral $I(\lambda)$ for any value of $\lambda$ and in particular for $\lambda \rightarrow \infty$. In this limit, the functional integral localizes onto the critical points of the functional $S^{Q}:=Q V$ which we refer to as the localizing solutions. The localizing solutions in general have both bosonic and fermionic collective coordinates.

One can choose

$$
V=(Q \Psi, \Psi)
$$

where $\Psi$ are the fermionic coordinates with some positive definite inner product defined on the fermions. In this case, the bosonic part of $S^{Q}$ can be written as a perfect square $(Q \Psi, Q \Psi)$, and hence critical points of $S^{Q}$ are the same as the critical points of $Q$. Let us denote this set of critical points of $Q$ by $\mathcal{M}_{Q}$. The reasoning above shows that the integral over the supermanifold $\mathcal{M}$ localizes to an integral over the submanifold $\mathcal{M}_{Q}$. In the large $\lambda$ limit, the integration for directions transverse can be performed exactly in the saddle point evaluation. One is then left with an integral over the submanifold $\mathcal{M}_{Q}$

$$
I=\int_{\mathcal{M}_{Q}} d \mu_{Q} h e^{-\mathcal{S}}
$$

with a measure $d \mu_{Q}$ induced on the submanifold by the original measure.

In our case in section $5, \mathcal{M}$ is the field space of off-shell supergravity, $\mathcal{S}$ is the off-shell supergravity action with appropriate boundary terms, $h$ is the supersymmetric Wilson line, $Q$ is a specific supercharge described in section $\mathrm{A}$ and section 5 , and $\Psi$ are all fermionic fields of the theory. We will find that the submanifold $\mathcal{M}_{Q}$ of localizing solutions is a family of nontrivial instantons as exact solutions to the equations of motion that follow from extremization of $S^{Q}$ labeled by $n_{v}+1$ real parameters $\left\{C^{I} ; I=0, \ldots, n_{v}\right\}$.

\subsection{Superconformal symmetries of the near horizon geometry}

The near-horizon geometry of a supersymmetric black hole in four dimensions is $A d S_{2} \times S^{2}$. After Euclidean continuation, the metric is

$$
d s^{2}=v\left[\left(r^{2}-1\right) d \theta^{2}+\frac{d r^{2}}{r^{2}-1}\right]+v\left[d \psi^{2}+\sin ^{2}(\psi) d \phi^{2}\right] .
$$


We have taken the radius $v$ of the $A d S_{2}$ factor to be the same as the radius of the $S^{2}$ factor which is a consequence of supersymmetry. There are several other coordinates that are useful. Substituting $r=\cosh (\eta)$, the metric takes the form

$$
d s^{2}=v\left[d \eta^{2}+\sinh ^{2}(\eta) d \theta^{2}\right]+v\left[d \psi^{2}+\sin ^{2}(\psi) d \phi^{2}\right] .
$$

One can also choose the stereographic coordinates

$$
w=\tanh \left(\frac{\eta}{2}\right) e^{i \theta}:=\rho e^{i \theta}, \quad z=\tan \left(\frac{\psi}{2}\right) e^{i \phi},
$$

in which the metric takes the form

$$
d s^{2}=v \frac{4 d w d \bar{w}}{(1-w \bar{w})^{2}}+v \frac{4 d z d \bar{z}}{(1+z \bar{z})^{2}} .
$$

Note that the interval for the coordinates are $1 \leq r<\infty$ and $0 \leq \eta<\infty$, and $0 \leq \rho<1$. In the $\omega$ coordinates, Euclidean $A d S_{2}$ can be readily recognized as the Poincaré disk with $\rho$ as the radial coordinate of the disk and a boundary at $\rho=1$.

The Weyl tensor for the metric (3.8) is zero and hence this metric is conformally flat. For later use it will useful to know this conformal transformation. To map we first map the Poincaré disk to the upper half plane by the transformation

$$
u=x+i y, \quad u=i \frac{1-i w}{1+i w} .
$$

The metric (3.7) in the new coordinates becomes

$$
d s^{2}=\frac{d x^{2}+d y^{2}+y^{2} d \Omega_{2}^{2}}{y^{2}}
$$

with $-\infty<x<+\infty$ and $0 \leq y<\infty$. From the above equation, we see that $A d S_{2} \times S^{2}$ is conformally flat. We also know that $\mathbb{R}^{4}$ is conformal to $S^{4}$ so it would be useful to compute the conformal factor relating $A d S_{2} \times S^{2}$ to $S^{4}$. In the $(\eta, \theta)$ coordinates we have the following conformal rescaling

$$
d s^{2}\left(A d S_{2} \times S^{2}\right)=\cosh ^{2}(\eta) d s^{2}\left(S^{4}\right) .
$$

Note that the conformal factor diverges at the boundary. Under a Weyl transformation

$$
g_{\mu \nu} \rightarrow e^{2 \Omega} g_{\mu \nu},
$$

a field with Weyl weight $a$ transforms as

$$
\Phi \rightarrow e^{-a \Omega} \Phi .
$$

Hence, such a field in the conformal frame with $A d S_{2} \times S^{2}$ metric will be mapped to the field in the conformal frame with $S^{4}$ metric by

$$
\Phi_{\mathrm{AdS}_{2} \times \mathrm{S}^{2}}=\frac{\Phi_{S^{4}}}{\cosh (\eta)^{a}} .
$$

This transformation will be useful later in section 5 . 
The superconformal symmetry of the near horizon geometry is the semidirect product $\mathrm{SU}(1,1 \mid 2) \rtimes \mathrm{SU}(2)^{\prime}$. The invariant subgroup $\mathrm{SU}(1,1 \mid 2)$ will be of our main interest which contains the bosonic subgroup $\mathrm{SU}(1,1) \times \mathrm{SU}(2)$. The first factor can be identified with the conformal symmetry of $A d S_{2}$ and is generated by $\left\{L, L_{ \pm}\right\}$. The second factor can be identified with the rotational symmetry of $S^{2}$ and is generated by $\left\{J, J_{ \pm}\right\}$. The factor $\mathrm{SU}(2)^{\prime}$ originates from the R-symmetry of $\mathcal{N}=2$ supergravity in four dimensions. The odd elements of the superalgebra are the superconformal symmetries $G_{r}^{i a}$. The commutations relations are

$$
\begin{aligned}
{\left[L, L_{ \pm}\right] } & = \pm L_{ \pm}, & {\left[L_{+}, L_{-}\right] } & =-2 L, \\
{\left[J, J^{ \pm}\right] } & = \pm J^{ \pm}, & {\left[J^{+}, J^{-}\right] } & =2 J, \\
{\left[L, G_{ \pm}^{i a}\right] } & = \pm \frac{1}{2} G_{ \pm}^{i a}, & {\left[L_{ \pm}, G_{\mp}^{i a}\right] } & =-i G_{ \pm}^{i a}, \\
{\left[J, G_{r}^{i \pm}\right] } & = \pm \frac{1}{2} G_{r}^{i \pm}, & {\left[J^{ \pm}, G_{r}^{i \mp}\right] } & =G_{r}^{i \pm}, \\
\left\{G_{+}^{i \pm}, G_{-}^{j \pm}\right\} & = \pm 4 \epsilon^{i j} J^{ \pm}, & \left\{G_{ \pm}^{i+}, G_{ \pm}^{j-}\right\} & =\mp 4 i \epsilon^{i j} L_{ \pm}, \\
\left\{G_{ \pm}^{i+}, G_{\mp}^{j-}\right\} & =4 \epsilon^{i j}(L \mp J) ; & \epsilon^{+-} & =-\epsilon-+=1 .
\end{aligned}
$$

Explicit expressions for the Killing spinors corresponding to these superconformal supersymmetries will be obtained in section A and will be required for localization in section 5 .

It is easy to see from the algebra that the generator $Q=G_{+}^{++}+G_{-}^{--}$squares to $4(L-J)$. Since $L$ is the generator of rotations of the Poincare disk and $J$ is the generator of rotations of $S^{2}$, the square $Q^{2}$ is the generator of a compact bosonic symmetry. This is the generator that we will use for localization.

\section{Off-shell formulation of the theory}

In this section, we review the off-shell formulation of supergravity due to [10-12]. This formalism has several attractive features.

1. First, it allows the supersymmetry transformations to be realized in an off-shell manner which will be crucial for us to apply localization to the functional integral for quantum entropy.

2. Second, one can also include within the formalism a class of curvature squared corrections to the theory that are encoded in the Weyl multiplet. This has made it possible to study the higher derivative corrections to supersymmetric black holes using the full power of supersymmetry for solving BPS equations in the classical theory.

3. Third, in the off-shell formalism, the supersymmetry transformations are specified once and for all and do not need to be modified as one modifies the action with higher derivative terms. This is analogous to the situation for diffeomorphisms where the transformation properties of the metric, for example, are specified once and for all and does not depend on the form the action. Since the localization action that we use is constructed using these supersymmetry transformations, the localizing solutions 
that we will obtain by minimizing this action will therefore be universal and not dependent on the form of the physical action. This is clearly greatly advantageous both at the technical and conceptual level.

In this section we rederive the classical properties of the black hole in this new language. This section is meant to set the stage and fix all the notations for the quantum calculation which we discuss in section 5 . It will therefore be concise; a detailed account of the off-shell formalism can be found, for example, in the review [45].

We use the conformal supergravity approach to $\mathcal{N}=2$ off-shell supergravity in four dimensions developed using superconformal multiplet calculus. The main idea is to extend the symmetries of the $\mathcal{N}=2$ Poincaré supergravity to the $\mathcal{N}=2$ superconformal algebra. This bigger algebra has dilatations, special conformal transformations, conformal $S$-supersymmetries, and local $\mathrm{SU}(2) \times \mathrm{U}(1)$ symmetries as extra symmetries compared to the Poincaré group. ${ }^{7}$ The conformal supergravity is then constructed as a gauge theory of this extended symmetry group.

Upon gauge fixing the extra superconformal symmetries, one gets the Poincaré supergravity. In this sense, they are both gauge equivalent. However, the multiplet structure of the superconformal supergravity is smaller and simpler than the Poincaré theory. The form of the supersymmetry transformation rules is also simpler in the superconformal formalism, and one has a systematic way of deriving invariant Lagrangians. Following this approach, one gets an off-shell formulation of supergravity coupled to vector multiplets.

In section 4.1, we first list the multiplets of the superconformal theory that will enter the theories we consider. In appendix B, we summarize some relevant aspects of the superconformal multiplet calculus including the supersymmetry variations of the various multiplets listed below. In section 4.2 we discuss the invariant action of our interest.

\subsection{Superconformal multiplets}

Our conventions are as follows. In the Minkowski theory, all fermion fields below are represented by Majorana spinors. In the Euclidean theory, they will be symplectic-Majorana. Greek indices $\mu, \nu, \ldots$ indicate the curved spacetime, latin indices $a, b, \ldots$ indicate the flat tangent space indices, and $i, j, \ldots$ denote the $\mathrm{SU}(2)$ index. The $\mathrm{SU}(2)$ indices are raised and lowered by complex conjugation. $A^{-} \equiv \varepsilon_{i j} A^{i j}$ for any $\mathrm{SU}(2)$ tensor $A^{i j}$. We will also use the superscript \pm to denote (anti) self-duality in spacetime, the conventions should be clear from the context. We use the covariant derivative $D_{a}$, which is defined to be covariant with respect to all the superconformal transformations as well as gauge fields of the theory if present. We also use The bosonic covariant derivative $\nabla_{a}$ is defined to be covariant with respect to all the bosonic transformations and the gauge fields, except the special conformal transformation.

We now summarize the field content of various multiplets.

\footnotetext{
${ }^{7}$ Note that the extra superconformal symmetry of this formalism is a gauge symmetry, not to be confused with the physical superconformal algebra of the near-horizon geometry of extremal black holes discussed in section 3.2 which is generated by the Killing vectors and Killing spinors of the background.
} 
1. Weyl multiplet: This is the gravity multiplet which contains all gauge fields arising from gauging the full superconformal symmetries. The field content is:

$$
\mathbf{w}=\left(e_{\mu}^{a}, w_{\mu}^{a b}, \psi_{\mu}^{i}, \phi_{\mu}^{i}, b_{\mu}, f_{\mu}^{a}, A_{\mu}, \mathcal{V}_{\mu j}^{i}, T_{a b}^{i j}, \chi^{i}, D\right) .
$$

The fields $\left(e_{\mu}^{a}, w_{\mu}^{a b}\right)$ are the gauge fields for translations (vielbien) and Lorentz transformations; $\psi_{\mu}^{i}, \phi_{\mu}^{i}$ are the gauge fields for Q-supersymmetries and the conformal S-supersymmetries; $\left(b_{\mu}, f_{\mu}^{a}\right)$ are the gauge fields for dilatations and the special conformal transformations; and $\left(\mathcal{V}_{\mu j}^{i}, A_{\mu}\right)$ are the gauge fields for the $\mathrm{SU}(2)$ and $\mathrm{U}(1)$ R-symmetries. Imposition of the 'conventional constraints' determines $w_{\mu}^{a b}, \phi_{\mu}^{i}, f_{\mu}^{a}$ in terms of other fields and one is left with $24+24$ independent degrees of freedom. The $\mathrm{SU}(2)$ doublet of Majorana spinors $\chi^{i}$, the antisymmetric anti self-dual auxiliary field $T_{a b}^{i j}$ and the real scalar field $D$ are all auxiliary fields, some of which will play a non-trivial role later. This multiplet contains the gravitational degrees of freedom.

2. Vector multiplet: The field content is

$$
\mathbf{X}^{I}=\left(X^{I}, \Omega_{i}^{I}, A_{\mu}^{I}, Y_{i j}^{I}\right)
$$

with $8+8$ degrees of freedom. $X^{I}$ is a complex scalar, the gaugini $\Omega_{i}^{I}$ are an $\mathrm{SU}(2)$ doublet of chiral fermions, $A_{\mu}^{I}$ is a vector field, and $Y_{i j}^{I}$ are an $\mathrm{SU}(2)$ triplet of auxiliary scalars. This multiplet contains the gauge field degrees of freedom.

3. Chiral multiplet: The field content is

$$
\widehat{\mathbf{A}}=\left(\widehat{A}, \widehat{\Psi}_{i}, \widehat{B}_{i j}, \widehat{F}_{a b}^{-}, \widehat{\Lambda}_{i}, \widehat{C}\right)
$$

with $16+16$ components. $\widehat{A}, \widehat{C}$ are complex scalars, $\widehat{B}_{i j}$ is a complex $\mathrm{SU}(2)$ triplet, $\widehat{F}_{a b}^{-}$is an antiselfdual Lorentz tensor, and $\widehat{\Psi}_{i}, \widehat{\Lambda}_{i}$ are $\mathrm{SU}(2)$ doublets of left-handed fermions. The action will also contain the conjugated right handed multiplet. One can impose a supersymmetric constraint on the chiral multiplet to get a reduced chiral multiplet with $8+8$ degrees of freedom.

The covariant quantities of a vector multiplet are associated with a reduced chiral multiplet. The covariant quantities of the Weyl multiplet are also associated with a reduced chiral multiplet $\mathbf{W}_{a b}^{i j}$. Products of chiral multiplets are also chiral, and one thus gets a chiral multiplet $\widehat{\mathbf{A}}=\mathbf{W}^{2}=\varepsilon_{i k} \varepsilon_{j l} \mathbf{W}_{a b}^{i j} \mathbf{W}^{a b k l}$. The lowest component of $\widehat{\mathbf{A}}$ is $\widehat{A}=\left(T_{a b}^{i j} \varepsilon_{i j}\right)^{2}$ and the highest component of $\widehat{\mathbf{A}}$ contains terms quadratic and linear in the curvature. The problem of building Lagrangians with terms quadratic in the curvature thus reduces to the simpler problem of coupling the chiral multiplet $\widehat{\mathbf{A}}$ to the superconformal theory.

4. Compensating multiplet: This multiplet will be used as a compensator to fix the extra gauge transformations. There are three types of compensators that have been used in the literature so far, a non-linear multiplet, a compensating hypermultiplet and a tensor multiplet. As an example, we discuss the non-linear multiplet [45, 46]. Other 
multiplets have their relative advantages, in particular the compensating hypermultiplet is used extensively for the treatment of higher derivative terms [47].

Non-linear multiplet:

$$
\left(\Phi_{\alpha}^{i}, \lambda^{i}, M^{i j}, V_{a}\right)
$$

where $\lambda^{i}$ is a spinor $\mathrm{SU}(2)$ doublet, $M^{i j}$ is a complex antisymmetric matrix of Lorentz scalars, and $V_{a}$ is a real Lorentz vector. $\Phi_{\alpha}^{i}$ is an $\mathrm{SU}(2)$ matrix of scalar fields with the $\alpha$ index transforming in the fundamental of a rigid $\mathrm{SU}(2)$, it describes three real scalars. Naively, the multiplet has $9+8$ degrees of freedom, but there is a supersymmetric constraint on the vector $V_{a}$ which reduces the degrees of freedom to $8+8:$

$$
D^{a} V_{a}-3 D-\frac{1}{2} V^{a} V_{a}-\frac{1}{4}\left|M_{i j}\right|^{2}+D^{a} \Phi^{i}{ }_{\alpha} D_{a} \Phi^{\alpha}{ }_{i}+\text { fermions }=0
$$

\subsection{Superconformal action}

The procedure to get invariant actions is as follows: one first finds an invariant Lagrangian for a chiral multiplet, this was solved in [48]. The second step is to write down a scalar function, the prepotential $F\left(X^{I}\right)$ of the vector multiplets which is a meromorphic homogeneous function of weight 2. One then uses the chiral Lagrangian of the first step for the chiral multiplet $\mathbf{F}$. This gives the two derivative $\mathcal{N}=2$ Poincaré supergravity after gauge fixing. To include coupling to curvature square terms, one extends the function $F$ to depend on the lowest component $\widehat{A}$ of the chiral multiplet $\widehat{\mathbf{A}}=\mathbf{W}^{2} . F\left(X^{I}, \widehat{A}\right)$ is holomorphic and homogeneous of degree two in all its variables. One then uses the chiral Lagrangian of the first step for the chiral multiplet $\mathbf{F}$.

We use the following notations. The prepotential which is a meromorphic function of its arguments obeys the homogeneity condition:

$$
F\left(\lambda X, \lambda^{2} \widehat{A}\right)=\lambda^{2} F(X, \widehat{A}) .
$$

Its various derivatives are defined as:

$$
F_{I}=\frac{\partial F}{\partial X^{I}}, \quad F_{\widehat{A}}=\frac{\partial F}{\partial \widehat{A}}, \quad F_{I J}=\frac{\partial^{2} F}{\partial X^{I} \partial X^{J}}, \quad F_{\widehat{A} I}=\frac{\partial^{2} F}{\partial X^{I} \partial \widehat{A}}, \quad F_{\widehat{A} \widehat{A}}=\frac{\partial^{2} F}{\partial \widehat{A}^{2}} .
$$

Following the above procedure, one gets a invariant action for $I=1,2, \ldots, N_{V}+1$ vectors coupled to conformal supergravity. The bosonic part of the action is:

$$
\begin{aligned}
e^{-1} \mathcal{L}= & i\left[\bar{F}_{I} X^{I}\left(\frac{1}{6} R-D\right)+\nabla_{\mu} F_{I} \nabla^{\mu} \bar{X}^{I}\right. \\
& +\frac{1}{4} F_{I J}\left(F_{a b}^{-I}-\frac{1}{4} \bar{X}^{I} T_{a b}^{i j} \varepsilon_{i j}\right)\left(F^{-a b J}-\frac{1}{4} \bar{X}^{J} T_{a b}^{i j} \varepsilon_{i j}\right)-\frac{1}{8} F_{I}\left(F_{a b}^{+I}-\frac{1}{4} X^{I} T_{a b i j} \varepsilon^{i j}\right) T_{a b}^{i j} \varepsilon_{i j} \\
& -\frac{1}{8} F_{I J} Y_{i j}^{I} Y^{J i j}-\frac{1}{32} F\left(T_{a b i j} \varepsilon^{i j}\right)^{2} \\
& +\frac{1}{2} F_{\widehat{A}} \widehat{C}-\frac{1}{8} F_{\widehat{A} \widehat{A}}\left(\varepsilon^{i k} \varepsilon^{j l} \widehat{B}_{i j} \widehat{B}_{k l}-2 \widehat{F}_{a b}^{-} \widehat{F}_{a b}^{-}\right)+\frac{1}{2} \widehat{F}^{-a b} F_{\widehat{A} I}\left(F_{a b}^{-I}-\frac{1}{4} \bar{X}^{I} T_{a b}^{i j} \varepsilon_{i j}\right) \\
& \left.-\frac{1}{4} \widehat{B}_{i j} F_{\widehat{A} I} Y^{I i j}\right]+ \text { h.c. }
\end{aligned}
$$


To get to the $\mathcal{N}=2$ Poincaré supergravity, one has to gauge fix the extra gauge transformations of the superconformal theory. To gauge fix the special conformal transformations, one sets the K-gauge:

$$
b_{\mu}=0
$$

To gauge fix the dilatations, one impose the D-gauge:

$$
-i\left(X^{I} \bar{F}_{I}-F_{I} \bar{X}^{I}\right)=1 .
$$

To fix the chiral U(1) symmetry, one fixes the A-gauge:

$$
X^{0}=\bar{X}^{0}
$$

Due to these constraints on the scalars, the Poincaré supergravity has only $N_{V}$ independent scalars.

In order to fix the $S$-supersymmetry, one imposes another gauge called the $S$-gauge. This constraint can be solved by eliminating one of the vector multiplet fermions. This gauge also breaks $Q$-supersymmetry, but a combination of the $S$ and $Q$ supersymmetries is preserved and corresponds to the physical supertransformations in the Poincaré theory.

Finally, to fix the local $\mathrm{SU}(2)$ symmetry, one imposes the $V$-gauge:

$$
\Phi_{\alpha}^{i}=\delta_{\alpha}^{i}
$$

At each step in the gauge fixing process, one has to be careful to respect the previous gauge choices, and this leads to compensating field dependent transformations in the rules for the various remaining transformations. This is one of the reasons the final theory is more complicated. Finally, one has to solve algebraic equations to get rid of the auxiliary fields $D$ and $\chi$. At the end of this procedure, one gets the $\mathcal{N}=2$ Poincaré supergravity with a bosonic Lagrangian:

$$
\begin{aligned}
8 \pi e^{-1} \mathcal{L}= & \left(-i\left(X^{I} \bar{F}_{I}-F_{I} \bar{X}^{I}\right)\right) \cdot\left(-\frac{1}{2} R\right) \\
& +\left[i \nabla_{\mu} F_{I} \nabla^{\mu} \bar{X}^{I}+\frac{1}{4} i F_{I J}\left(F_{a b}^{-I}-\frac{1}{4} \bar{X}^{I} T_{a b}^{i j} \varepsilon_{i j}\right)\left(F^{-a b J}-\frac{1}{4} \bar{X}^{J} T_{a b}^{i j} \varepsilon_{i j}\right)\right. \\
& -\frac{1}{8} i F_{I}\left(F_{a b}^{+I}-\frac{1}{4} X^{I} T_{a b i j} \varepsilon^{i j}\right) T_{a b}^{i j} \varepsilon_{i j} \\
& -\frac{1}{8} i F_{I J} Y_{i j}^{I} Y^{J i j}-\frac{i}{32} F\left(T_{a b i j} \varepsilon^{i j}\right)^{2} \\
& +\frac{1}{2} i F_{\widehat{A}} \widehat{C}-\frac{1}{8} i F_{\widehat{A} \widehat{A}}\left(\varepsilon^{i k} \varepsilon^{j l} \widehat{B}_{i j} \widehat{B}_{k l}-2 \widehat{F}_{a b}^{-} \widehat{F}_{a b}^{-}\right)+\frac{1}{2} i \widehat{F}^{-a b} F_{\widehat{A} I}\left(F_{a b}^{-I}-\frac{1}{4} \bar{X}^{I} T_{a b}^{i j} \varepsilon_{i j}\right) \\
& \left.-\frac{1}{4} i \widehat{B}_{i j} F_{\widehat{A} I} Y^{I i j}+\text { h.c. }\right] \\
& -i\left(X^{I} \bar{F}_{I}-F_{I} \bar{X}^{I}\right) \cdot\left(\nabla^{a} V_{a}-\frac{1}{2} V^{a} V_{a}-\frac{1}{4}\left|M_{i j}\right|^{2}+D^{a} \Phi^{i}{ }_{\alpha} D_{a} \Phi^{\alpha}{ }_{i}\right) .
\end{aligned}
$$


Note that both the covariant derivatives defined above are used in this expression, they are related by

$$
D^{a} V_{a}=\nabla^{a} V_{a}-2 f_{a}^{a}+\text { fermionic terms . }
$$

\section{Localization}

We now turn to the evaluation of the supersymmetric black hole functional integral defined in section 2 using the localization techniques discussed in section 3. We use the formalism of section 4 so that the supercharge used for localization is realized off-shell.

The on-shell equations of motion that follow from the above Lagrangian (4.13) admit a half-BPS black hole solution [47, 49-51]. The near horizon geometry is an $A d S_{2} \times S^{2}$ which admits eight conformal supersymmetries. ${ }^{8}$ The values of other fields are determined by the attractor mechanism [52-54] in terms of the charges consistent with the isometries. The near-horizon $A d S_{2} \times S^{2}$ geometry with the attractor values of the other fields can also directly be derived from the BPS equations [55].

We first review this on-shell solution in section 5.1. We then proceed to find the localizing instanton solution in section 5.2 and evaluate the renormalized action for this solution in section 5.3. We will sometimes refer to the localizing solution as the off-shell solution since for this solution the scalar fields are excited away from the attractor values inside the $A d S_{2}$. In section 5.4 we put together these ingredients to reduce the functional integral of $\hat{W}(q, p)$ to an ordinary integral on the localizing submanifold.

\section{$5.1 \quad$ On-shell attractor geometry}

Symmetries of $A d S_{2} \times S^{2}$ imply that various field in the near horizon region take the form

$$
\begin{aligned}
d s^{2} & =v\left[-\left(r^{2}-1\right) d t^{2}+\frac{d r^{2}}{r^{2}-1}\right]+v\left[d \psi^{2}+\sin ^{2}(\psi) d \phi^{2}\right], \\
F_{r t}^{I} & =e_{*}^{I}, \quad F_{\psi \phi}^{I}=p^{I} \sin \psi, \quad X^{I}=X_{*}^{I}, \quad T_{r t}^{-}=v w, \\
D-\frac{1}{3} R & =0, \quad M_{i j}=0, \quad \Phi_{i}^{\alpha}=\delta_{i}^{\alpha}, \quad Y_{i j}^{I}=0 .
\end{aligned}
$$

The values of the constants $\left(e_{*}^{I}, X_{*}^{I}, v_{*}\right)$ that appear in this solution are determined in terms of the charges $\left(q_{I}, p^{I}\right)$ by the attractor equations which follow from the BPS conditions [49], or, equivalently using the entropy function formalism [46]:

$$
\begin{aligned}
v=\frac{16}{\bar{w} w}, \quad \hat{A} & =-4 \omega^{2}, \\
e_{*}^{I}-i p^{I}-\frac{1}{2} \bar{X}_{*}^{I} v w & =0, \\
4 i\left(\bar{w}^{-1} \bar{F}_{I}-w^{-1} F_{I}\right) & =q_{I} .
\end{aligned}
$$

\footnotetext{
${ }^{8}$ As mentioned above, these conformal supersymmetries are not the conformal supersymmetries of the four-dimensional theory discussed in the last section, the latter are gauge symmetries in that formalism.
} 
Taking the real and imaginary parts of (5.3) and substituting (5.2) gives

$$
\begin{aligned}
4\left(\bar{w}^{-1} \bar{X}_{*}^{I}+w^{-1} X_{*}^{I}\right) & =e_{*}^{I}, \\
4 i\left(\bar{w}^{-1} \bar{X}_{*}^{I}-w^{-1} X_{*}^{I}\right) & =p^{I},
\end{aligned}
$$

where $F_{I}$ should be thought of as functions of $X_{*}^{I}$. This geometry preserves eight superconformal supersymmetries as reviewed section A which extends the symmetries to the supergroup $\mathrm{SU}(1,1 \mid 2) \rtimes \mathrm{SU}(2)^{\prime}$ discussed in section 3.2. The field $w$ can be fixed by a gauge choice. In the rest of the paper, we choose a gauge in which $w=\bar{w}=4$ using the local scaling symmetry of the Lagrangian and the $U(1)$ invariance. In this gauge, the radius $v$ of both $A d S_{2}$ and $S^{2}$ equals one, this simplifies the discussion of Killing spinors. ${ }^{9}$

\subsection{Localizing action and the localizing instantons}

In order to use the technique of localization for our system, we need to pick a subalgebra of the full supersymmetry algebra discussed in section 3.2, whose bosonic generator is compact. We shall choose the subalgebra generated by the action of the supercharge

$$
Q_{1}=G_{+}^{++}+G_{-}^{--}
$$

which generates the compact $\mathrm{U}(1)$ action:

$$
Q_{1}^{2}=4(L-J)
$$

The explicit form of the Killing spinors can be found in section A. The above choice of the supercharge corresponds to choosing the supersymmetry parameter $\zeta_{1}$ defined in (A.17). In this section, we use the notation $Q \equiv Q_{1}, \zeta \equiv \zeta_{1}$.

The localizing Lagrangian is then defined by

$$
\mathcal{L}^{Q}:=Q V \quad \text { with } \quad V:=(Q \Psi, \Psi),
$$

where $\Psi$ refers to all fermions in the theory. The localizing action is then defined by

$$
S^{Q}=\int d^{4} x \sqrt{g} \mathcal{L}^{Q}
$$

The localization equations that follow from this action are

$$
Q \Psi=0
$$

These are the equations that we would like to solve.

We assume that the supergroup isometries of the near horizon geometry are not broken further by the Weyl multiplet fields. By construction, as long as these symmetries are maintained, the fermions of the Weyl multiplet do not transform under the action of $Q$ (A.1)-(A.3) in the $A d S_{2}$ attractor background. One can check that the fermions of the

\footnotetext{
${ }^{9}$ This is different from the gauge used in the previous section and also from the gauge $\omega=8$ which is commonly used. These gauge choices do not affect considerations in this paper, but a better understanding of different gauge choices can be useful to simplify the analysis. We plan to return to this issue in future.
} 
chiral multiplet and the non-linear multiplet also do not transform in this background. This prompts us to look for solutions where one still has the $A d S_{2}$ attractor geometry, but the scalars of the vector multiplets can move away from their attractor values. ${ }^{10}$ As we will see there do exist nontrivial solutions where the vector multiplet fields get excited maintaining the symmetries of the attractor geometry.

The action of $Q$ on the fermionic field of the vector multiplet takes the form

$$
\begin{aligned}
Q \Omega_{+}^{I i} & =\frac{1}{2}\left(F_{\mu \nu}^{I-}-\frac{1}{4} \bar{X}^{I} T_{\mu \nu}^{-}\right) \gamma^{\mu} \gamma^{\nu} \zeta_{+}^{i}+2 i \not \partial X^{I} \zeta_{-}^{i}+Y_{j}^{I i} \zeta_{+}^{j}, \\
Q \Omega_{-}^{I i} & =\frac{1}{2}\left(F_{\mu \nu}^{I+}-\frac{1}{4} X^{I} T_{\mu \nu}^{+}\right) \gamma^{\mu} \gamma^{\nu} \zeta_{-}^{i}+2 i \not \partial \bar{X}^{I} \zeta_{+}^{i}+Y_{j}^{I i} \zeta_{-}^{j} .
\end{aligned}
$$

Let us recall the attractor equations for the constant values of the various fields in terms of the electric gauge field strengths $e^{I}$ and the magnetic charges $p^{I}$ :

$$
e_{*}^{I}-i p^{I}-2 \bar{X}_{*}^{I}=0, \quad e_{*}^{I}+i p^{I}-2 X_{*}^{I}=0 \quad Y_{i j *}^{I}=0 .
$$

We are interested in the off-shell solutions in which the vector multiplet scalars $X^{I}$ move away from their attractor values $X_{*}^{I}$. We therefore parametrize the off-shell $X^{I}$ fields as

$$
X^{I}:=X_{*}^{I}+\Sigma^{I}, \quad \bar{X}^{I}:=\bar{X}_{*}^{I}+\bar{\Sigma}^{I},
$$

so that $\Sigma^{I}$ and $\bar{\Sigma}^{I}$ are values the scalar fields away from the attractor values. We further write

$$
\Sigma^{I}=H^{I}+i J^{I}, \quad \bar{\Sigma}^{I}=H^{I}-i J^{I} .
$$

Note that $Y_{i j}^{I}$ are triplets under the $\mathrm{SU}(2)$ rotation. It will turn out that for the BPS equations that we solve, they all have to be aligned along the same direction in the $\mathrm{SU}(2)$ space. Hence we parametrize them as

$$
Y_{1}^{I 1}=-Y_{2}^{I 2}=K^{I} ; \quad Y_{2}^{I 1}=Y_{1}^{I 2}=0 .
$$

Similarly we parametrize the gauge fields away from the attractor values as

$$
F_{\mu \nu}^{I}=F_{\mu \nu *}^{I}+f_{\mu \nu}^{I} .
$$

With this parametrization, we can add the two equations (5.12) and perform a Euclidean continuation to obtain

$$
Q \Omega^{I i}=\frac{1}{2} f_{a b}^{I} \gamma^{a} \gamma^{b} \zeta^{i}+2 i \not \partial H^{I} \zeta^{i}+2 \not \partial J^{I} \gamma_{5} \zeta^{i}-2 i H^{I} \gamma^{0} \gamma^{1} \zeta^{i}-2 J^{I} \gamma^{2} \gamma^{3} \zeta^{i}+Y_{j}^{I i} \zeta^{j} .
$$

for the Dirac spinors $\Omega^{I i}=\Omega_{+}^{I i}+\Omega_{-}^{I i}$. Note that the $a, b$ are tangent space indices and all gamma matrices $\gamma^{a}$ above are constant matrices of Euclidean $\mathbb{R}^{4}$.

\footnotetext{
${ }^{10}$ Solutions more general than our simplifying ansatz are in principle possible where the Weyl multiplet fields also vary inside the $A d S_{2}$.
} 
The inner product for spinors $\chi_{1}$ and $\chi_{2}$ in Euclidean space is simply

$$
\left(\chi_{1}, \chi_{2}\right)=\chi_{1}^{\dagger} \chi_{2}
$$

With this inner product, the localization Lagrangian (5.9) restricted to only the vector multiplet fermions is given by

$$
\mathcal{L}^{Q}=Q V:=Q(Q \Psi, \Psi)
$$

with $V$ chosen as in (5.9) with $\Psi$ denoting the vector multiplet fermions. Note that $V$ is $\mathrm{H}$-invariant because $\zeta$ is independent of the combination $\theta-\phi$ and $H$ is the vector field that generates translations along $\theta-\phi$. The bosonic part of this Lagrangian is

$$
\left.\mathcal{L}_{\text {bos }}^{Q} \equiv Q V\right|_{\text {bosonic }}=\sum_{I=0}^{n_{V}}\left(Q \Omega^{I}, Q \Omega^{I}\right) \text {. }
$$

With our choice of the inner product (5.20) this Lagrangian is manifestly positive definite.

The choice of $Q$ is determined by the choice of the Killing spinor $\zeta$. Substituting the explicit form of the Killing spinor $\zeta$ and the gamma matrices defined in section A, the bosonic Lagrangian $\mathcal{L}_{\text {bos }}^{Q}$ as a function of the fields $H, J, K, f$ can be evaluated after somewhat tedious algebra. We find that $\frac{1}{2} \mathcal{L}_{\text {bos }}^{Q}$ equals

$$
\begin{aligned}
& \cosh (\eta)[K-2 \operatorname{sech}(\eta) H)]^{2} \\
& +4 \cosh (\eta)\left[H_{1}+H \tanh (\eta)\right]^{2}+4 \cosh (\eta)\left[H_{0}^{2}+H_{2}^{2}+H_{3}^{2}\right] \\
& +2 A\left[f_{01}^{-}-J-\frac{1}{A}\left(\sin (\psi) J_{3}-\sinh (\eta) J_{1}\right)\right]^{2}+2 B\left[f_{01}^{+}+J-\frac{1}{B}\left(\sin (\psi) J_{3}+\sinh (\eta) J_{1}\right)\right]^{2} \\
& +2 A\left[f_{03}^{-}+\frac{1}{A}\left(\sin (\psi) J_{1}+\sinh (\eta) J_{3}\right)\right]^{2}+2 B\left[f_{03}^{+}+\frac{1}{B}\left(\sin (\psi) J_{1}-\sinh (\eta) J_{3}\right)\right]^{2} \\
& +2 A\left[f_{02}^{-}+\frac{1}{A}\left(\sin (\psi) J_{0}+\sinh (\eta) J_{2}\right)\right]^{2}+2 B\left[f_{02}^{+}-\frac{1}{B}\left(\sin (\psi) J_{0}+\sinh (\eta) J_{2}\right)\right]^{2} \\
& +\frac{4 \cosh (\eta)}{A B}\left[\sinh (\eta) J_{0}-\sin (\psi) J_{2}\right]^{2}+\frac{4 \cosh (\eta) \sinh ^{2}(\eta)}{A B}\left[J_{1}^{2}+J_{3}^{2}\right],
\end{aligned}
$$

where

$$
H_{a}^{I}:=e_{a}^{\mu} \partial_{\mu} H^{I}, \quad J_{a}^{I}:=e_{a}^{\mu} \partial_{\mu} J^{I}
$$

and

$$
A:=\cosh (\eta)+\cos (\psi), \quad B:=\cosh (\eta)-\cos (\psi) .
$$

It is understood that in (5.23) all squares are summed over the index $I$. Recall that $a=0,1,2,3$ correspond to the directions along the coordinates $\theta, \eta, \phi, \psi$ respectively used for example in (3.8). Since $A$ and $B$ are positive, $\mathcal{L}_{\text {bos }}^{Q}$ is a sum of positive squares.

The minimization equations now follow by setting each of the squares in (5.23) to zero. This leads to simple first order differential equations for various fields which have to be solved with boundary conditions consistent with the definition of the original functional 
integral on Euclidean $A d S_{2}$ space. Equations (5.15), (5.17), (5.18) imply that fields $\Sigma^{I}$ and $K^{I}$ and $f^{I}$ must vanish at the boundary.

It is easy to see that with these boundary conditions, $J^{I}$ and $f_{a b}^{I}$ must both vanish throughout space. Setting the first line in (5.23) to zero implies

$$
K^{I}=\frac{2 H^{I}}{\cosh (\eta)}
$$

Setting the second line in (5.23) to zero leads to differential equations that can be easily solved to obtain

$$
H^{I}=\frac{C^{I}}{\cosh (\eta)}
$$

We have thus succeeded in finding a family of exact solutions to the localization equations which respect the classical boundary conditions on $A d S_{2}$ and are smooth everywhere in the interior. In terms of the original variables defined in (5.15), we have

$$
\begin{aligned}
X^{I} & =X_{*}^{I}+\frac{C^{I}}{\cosh (\eta)}, \quad \bar{X}^{I}=\bar{X}_{*}^{I}+\frac{C^{I}}{\cosh (\eta)} \\
Y_{1}^{I 1} & =-Y_{2}^{I 2}=\frac{2 C^{I}}{\cosh (\eta)^{2}} .
\end{aligned}
$$

Since the scalar fields are now excited away from their attractor values, they are no longer at the minimum of the classical entropy function. Even though scalar fields 'climb up' the potential away from the minimum of the entropy function the solution remains Qsupersymmetric (in the Euclidean theory) because an auxiliary field gets excited appropriately to satisfy the Killing spinor equations.

It is worth pointing out that the solutions (5.27) and (5.26) look much simpler if we use the conformal transformation (3.13) in section 3.2 to map $A d S_{2} \times S^{2}$ to $S^{4}$. Since the scalar fields $X$ and the auxiliary fields $Y$ have Weyl weight 1 and 2 respectively, and since the conformal factor is $\cosh (\eta)$, the fields $\Sigma$ and $Y$ are simply constant on $S^{4}$. This is very similar to the localizing solution found by Pestun [14] in a very different context of computing the expectation value of Wilson line in super Yang-Mills theory on $S^{4}$. Of course, under this conformal transformation the attractor values also will transform and since they are constant on $A d S_{2} \times S^{2}$, they will no longer be constant on $S^{4}$. It is therefore more natural to work in the $A d S_{2} \times S^{2}$ frame. In any case, for computing the quantum entropy, the $A d S_{2}$ boundary conditions play an important role as we will see in the next subsection. As pointed out in [9], in this frame our computation has close formal similarity with the gauge theory computation of 't Hooft-Wilson line in the formulation of $[56,57]$ which could be useful in the computation of one-loop determinants and the instanton contributions. Note that we are using localization techniques to evaluate a bulk functional integral of supergravity whereas in $[14,56,57]$ it was used to evaluate a functional integral in the boundary gauge theory. 


\subsection{Renormalized action for the localizing instantons}

To obtain the exact macroscopic quantum partition function we would like to evaluate the renormalized action restricted to the submanifold $\mathcal{M}_{Q}$ in field space of localizing instantons. We will find that even though both the original action and the solution are rather complicated, the renormalized action is a remarkably simple function of the collective coordinates $\left\{C^{I}\right\}$ determined entirely by the prepotential. Recall that the renormalized action defined in section 2 takes the form

$$
\mathcal{S}_{\text {ren }}:=\mathcal{S}_{\text {bulk }}+\mathcal{S}_{\text {bdry }}+i \frac{q_{I}}{2} \int_{0}^{2 \pi} A_{\theta}^{I} d \theta
$$

The charges used here are related to the ones used in (2.11) by $q_{I}=-2 q_{i}$ to be consistent with the normalization of gauge fields used in the literature, for example, in the reviews $[45,58]$.

We proceed to evaluate the bulk action given as a four dimensional integral of the the supergravity Lagrangian (4.13) over $A d S_{2} \times S^{2}$. We note first that since various auxiliary fields vanish for the off-shell solution, the Lagrangian (4.13) simplifies to (recall $\left.\widehat{A}=\left(T_{a b}^{i j} \varepsilon_{i j}\right)^{2}\right)$ :

$$
\begin{aligned}
8 \pi \mathcal{L}= & -\frac{i}{2}\left(X^{I} \bar{F}_{I}-\bar{X}^{I} F_{I}\right) R \\
& +\left[i \partial_{\mu} F_{I} \partial^{\mu} \bar{X}^{I}+\frac{i}{4} F_{I J}\left(F_{\mu \nu}^{-I}-\frac{1}{4} \bar{X}^{I} T_{\mu \nu}^{i j} \varepsilon_{i j}\right)\left(F^{-J \mu \nu}-\frac{1}{4} \bar{X}^{J} T^{\mu \nu i j} \varepsilon_{i j}\right)\right. \\
& \left.+\frac{i}{8} \bar{F}_{I}\left(F_{\mu \nu}^{-I}-\frac{1}{4} \bar{X}^{I} T_{\mu \nu i j} \varepsilon^{i j}\right) T_{\mu \nu}^{i j} \varepsilon_{i j}-\frac{i}{8} F_{I J} Y_{i j}^{I} Y^{J i j}+\frac{i}{32} \bar{F} \hat{A}+\frac{i}{2} F_{\hat{A}} \hat{C}+\text { h.c. }\right] .
\end{aligned}
$$

Moreover, for $A d S_{2} \times S^{2}$ both the Ricci scalar $R$ and the Weyl tensor $C$ are zero. Substituting $X^{I}=X_{*}^{I}+\Sigma^{I}$ and $\bar{X}^{I}=\bar{X}_{*}^{I}+\bar{\Sigma}^{I}$ from (5.15) and using the attractor equation (5.14) in the form

$$
F_{\mu \nu}^{-I}-\frac{1}{4}{\overline{X_{*}}}^{I} T_{\mu \nu}^{i j} \varepsilon_{i j}=0
$$

we get

$$
8 \pi \mathcal{L}=i F_{I J}\left(\partial_{\eta} \bar{\Sigma}^{I}\right)\left(\partial_{\eta} \Sigma^{J}\right)-i F_{I J} \bar{\Sigma}^{I} \Sigma^{J}+\frac{i}{4} F_{I J} K^{I} K^{J}+2 i \bar{F}_{I} \bar{\Sigma}^{I}-2 i \bar{F}+\text { h.c. } .
$$

Substituting the solution (5.28) into the above equation, we find that the first three terms of add up to zero. We are thus left with

$$
8 \pi \mathcal{L}=2 i \bar{F}_{I} \bar{\Sigma}^{I}-2 i \bar{F}+h . c . .
$$

Since we keep the classical values $X_{*}^{I}, \bar{X}_{*}^{I}$ fixed in this problem, differentiating with respect to $X^{I}$ is the same as differentiating with respect to $\Sigma^{I}$. This can be explicitly evaluated to find

$$
8 \pi \mathcal{L}=2 i \partial_{r}(r(F-\bar{F})), \quad \text { with } \quad \Sigma^{I}=\frac{C^{I}}{r}
$$


The $\mathcal{N}=2$ supergravity Euclidean action is

$$
\mathcal{S}_{\text {bulk }}=\int d^{4} x \sqrt{g} \mathcal{L}
$$

The off-shell fields do not depend on the coordinates of the $S^{2}$ and the angular variable $\theta$ of the $A d S_{2}$. These integrals can be done be done trivially and give an overall factor of $8 \pi^{2}$, so that

$$
\begin{aligned}
\mathcal{S}_{\text {bulk }} & =8 \pi^{2} \int_{0}^{\eta_{0}} \mathcal{L} \sinh (\eta) d \eta=8 \pi^{2} \int_{1}^{r_{0}} \mathcal{L} d r \\
& =2 \pi i \int_{1}^{r_{0}} d r \partial_{r}(r(F-\bar{F})) \\
& =2 \pi i r_{0}\left[F\left(X_{*}^{I}+\frac{C^{I}}{r_{0}}\right)-\bar{F}\left(X_{*}^{I}+\frac{C^{I}}{r_{0}}\right)\right]-2 \pi i\left[F\left(X_{*}^{I}+C^{I}\right)-\bar{F}\left(X_{*}^{I}+C^{I}\right)\right]
\end{aligned}
$$

The first piece in (5.39) which is linear in $r_{0}$ can be rewritten as:

$$
\begin{aligned}
2 \pi i r_{0} & \left(F\left(X_{*}^{I}+\frac{C^{I}}{r_{0}}\right)-\bar{F}\left(X_{*}^{I}+\frac{C^{I}}{r_{0}}\right)\right)= \\
& =2 \pi i r_{0}\left(F\left(X_{*}^{I}\right)-\bar{F}\left(X_{*}^{I}\right)\right)+2 \pi i\left(F_{I}\left(X_{*}^{I}\right)-\bar{F}_{I}\left(X_{*}^{I}\right)\right) C^{I}+\mathcal{O}\left(1 / r_{0}\right) \\
& =2 \pi i r_{0}\left(F\left(X_{*}^{I}\right)-\bar{F}\left(X_{*}^{I}\right)\right)-2 \pi q_{I} C^{I}+\mathcal{O}\left(1 / r_{0}\right)
\end{aligned}
$$

where we have used a Taylor expansion in the first line and the attractor equation

$$
F_{I}\left(X_{*}^{I}\right)-\bar{F}_{I}\left(X_{*}^{I}\right)=i q_{I}
$$

in the second.

The Wilson line evaluates to

$$
i \frac{q_{I}}{2} \int_{0}^{2 \pi} A_{\theta}^{I} d \theta=\pi q_{I} e_{*}^{I}\left(r_{0}-1\right)
$$

Hence we choose

$$
\mathcal{S}_{\text {bdry }}=-2 \pi r_{0}\left(\frac{q_{I} e_{*}^{I}}{2}+i\left(F\left(X_{*}^{I}\right)-\bar{F}\left(X_{*}^{I}\right)\right)\right) .
$$

so that $\mathcal{S}_{\text {ren }}=\mathcal{S}_{\text {bulk }}+\mathcal{S}_{\text {bdry }}+i \frac{q}{2} \oint A$ is finite.

As reviewed in section 2, the main purpose of the boundary action is to cancel the divergence in the bare bulk action plus Wilson line which grows linearly with the length of the boundary. In order to cancel this divergence, we use a boundary cosmological constant which must be specified along with the other boundary data. Indeed we have found that $\mathcal{S}_{\text {bdry }}$ which is a constant that grows linearly with the length of the boundary indeed only depends on the fixed charges and not on the fluctuating fields.

In general, however, there could be a finite part of the boundary action which does depend on the fields that are integrated over. The full boundary action should be constrained by supersymmetry. We shall discuss the supersymmetry of the functional integral 
in appendix $\mathrm{C}$. The conclusion of the analysis in appendix $\mathrm{C}$ is quite simple - the finite part of the boundary action in our problem actually vanishes due to supersymmetry, and therefore the above prescription for $S_{\text {ren }}$ as a sum of terms (5.37), (5.43) and (5.42) is already supersymmetric. In appendix $\mathrm{C}$, we shall rewrite the above in a manner that is manifestly supersymmetric. This rewriting takes the form of a functional integral with a supersymmetric Wilson line $[59,60]$ with the bulk action as above (5.37), and a boundary action which exactly cancels the boundary piece in (5.39).

We thus obtain the following expression for the renormalized action:

$$
\mathcal{S}_{\mathrm{ren}}=-\pi q_{I} e_{*}^{I}-2 \pi q_{I} C^{I}-2 \pi i\left(F\left(X_{*}^{I}+C^{I}\right)-\bar{F}\left(X_{*}^{I}+C^{I}\right)\right),
$$

The notation $e_{*}^{I}$ refers to the classical values of the electric field strengths as a function of the charges $\left(q_{I}, p^{I}\right)$. Using the scalar attractor values (5.5), and the new variable

$$
\phi^{I} \equiv e_{*}^{I}+2 C^{I},
$$

we can express the renormalized action in a remarkably simple form:

$$
\mathcal{S}_{\text {ren }}(\phi, q, p)=-\pi q_{I} \phi^{I}+\mathcal{F}(\phi, p)
$$

with

$$
\mathcal{F}(\phi, p)=-2 \pi i\left[F\left(\frac{\phi^{I}+i p^{I}}{2}\right)-\bar{F}\left(\frac{\phi^{I}-i p^{I}}{2}\right)\right] .
$$

Note that the electric field remains fixed at the attractor value but $\phi^{I}$ can still fluctuate with $C^{I}$ taking values over the real line. We will discuss the significance of this fact in section 6 . Note also that the prepotential is evaluated at precisely for values of the scalar fields at the origin of $A d S_{2}$ and not at the boundary of $A d S_{2}$. Thus the classical contribution to the localization integrand will be of the form

$$
e^{S_{\mathrm{ren}}}=e^{-\pi \phi^{I} q_{I}+\mathcal{F}(\phi, p)}
$$

There will be additional contribution to the integral which we discuss next.

\subsection{Evaluation of $\hat{W}(q, p)$}

We have thus determined which field configuration to integrate over and the classical action for these configuration. The full functional integral will require three additional ingredients.

- The integration measure over the $\left\{C^{I}\right\}$ fields over the submanifold $\mathcal{M}_{Q}$ of critical points of $Q$ simply descends from the measure $\mu$ of supergravity over the field space $\mathcal{M}$. We denote this measure by $[d C]_{\mu}$ which can be computed using standard methods of collective coordinate quantization.

- There will be one-loop determinants of fluctuations around the localizing manifold which can be evaluated from the quadratic piece of the localizing action $S^{Q}$. We denote this determinant contribution by $Z_{\text {det }}$. It is in principle a straightforward but technically involved computation. Very similar determinants have been analyzed 
in detail for gauge theory [14]. In string theory, the one-loop determinants and the duality invariance measure around the on-shell solution have been analyzed in [15] and in $[27,61]$ respectively. Some aspects of these computations both from gauge theory and from around the on-shell saddle point could be adapted to study the measure and determinants around our off-shell instantons solutions [16].

- In addition, there will be a contribution from point instantons and anti-instantons viewed as singular configurations that couple to the vector multiplet fields as long as they preserve the same supersymmetry. In gauge theory computations [14], the instantons will be localized at the center of $A d S_{2}$ and at the north pole of the $S^{2}$ whereas the anti-instantons will be be localized at the center of $A d S_{2}$ and at the south pole of the $S^{2}$. Since string theory contains gauge theory at low energies we expect a similar structure also in string theory. We denote this generating function for the instantons by $Z_{\text {inst }}$. The generating function for anti-instantons will be the complex conjugate of the generating function for instantons. We will thus get a factor of $\left|Z_{\text {inst }}\right|^{2}$ which will depend on the details of the string compactification, the spectrum of wrapped brane-instantons, and the duality frame under consideration. In gauge theory this generating function is the equivariant instanton partition function computed by Nekrasov [62]. Since the low energy limit of string theory will reduce to gauge theory on $A d S_{2} \times S^{2}$, it would be interesting to explore if there are generalization of the gauge theory results to string theory.

Putting these ingredients together we can conclude that the functional integral will have the form

$$
\hat{W}(q, p)=\int_{\mathcal{M}_{Q}} e^{-\pi \phi^{I} q_{I}} e^{\mathcal{F}(\phi, p)}\left|Z_{\text {inst }}\right|^{2} Z_{\operatorname{det}}[d C]_{\mu} .
$$

We have thus successfully reduced the functional integral to ordinary integrals. The dominant piece of the answer given by $e^{-S_{\mathrm{ren}}}$ we have already evaluated explicitly.

In specific string compactifications the undetermined factors $Z_{\text {det }}$ and $\left|Z_{\text {inst }}\right|^{2}$ can simplify. For example, with $\mathcal{N}=4$ supersymmetry, in gauge theory both $\left|Z_{\text {instanton }}\right|^{2}$ and $Z_{\text {det }}$ equal unity. Similarly, it was found in [15] that very similar determinant factors for vector multiplets equal unity $\mathcal{N}=4$ theories. One expects that this simplification will extend to the factors appearing in (5.49) around the localizing solution in $\mathcal{N}=4$ theories.

\section{Quantum entropy and the topological string}

We now turn to the original problem of evaluating of $W(q, p)$. There are several issues that have to be addressed to extend the supergravity computation to a full string computation.

- First, the full action of string theory of course contains more fields in addition to vector multiplets, in particular the hyper multiplets.

- Second, even if we restrict our attention to vector multiplets, the action will in general contain not just the F-terms which are chiral superspace integrals but also the Dterms which are nonchiral superspace integrals. 
- Third, there can be additional contributions from functional integral over orbifolds of $A d S_{2}$ that are allowed in the full string theory but not visible in supergravity.

We discuss these questions below.

\subsection{D-terms, hyper-multiplets, and evaluation of $W_{0}(q, p)$}

We have thus far considered only F-type terms for the action of the vector multiplets which are chiral integrals over $\mathcal{N}=2$ superspace of the form $\int d^{4} \theta$. The effective action of string theory will contain in general D-type terms which are nonchiral integrals over $\mathcal{N}=2$ superspace of the form $\int d^{4} \theta d^{4} \bar{\theta}$. It is not a priori clear that these terms will not contribute to the functional integral. We would like to make the following two observations in this connection.

- Since our localizing action $S^{Q}$ follows from off-shell supersymmetry transformations, it does not depend on what terms are present in the physical action $S$. Hence our localizing instanton solutions are universal and they will continue to exist even with the addition of the D-terms. The question then reduces to evaluating the D-terms on these solutions to obtain their contribution to the renormalized action.

- It has recently been shown [13] that a large class of D-type terms do not contribute to the Wald entropy. This class of terms are constructed using the 'kinetic multiplet' $T$ obtained from a chiral multiplet $\Phi$ of Weyl weight 0 by $T=\bar{D}^{4} \bar{\Phi}$ which transforms like a chiral multiplet of Weyl weight 2. One can construct now supersymmetry invariant terms in the action as chiral integrals $\int d^{4} \theta$ with arbitrary polynomials involving the kinetic multiplet and other chiral multiplets. Since four antichiral derivatives have the same effect as the four antichiral integrals, these terms correspond to D-terms with non chiral integrals $\int d^{4} \theta d^{4} \bar{\theta}$ of terms involving the original field $\Phi$. The nonrenormalization theorem of [13] shows that D-terms of this type do not contribute to the Wald entropy. Since the renormalized action of the localizing instantons follows from the bulk action and has the same form as the entropy function, it should be possible to extend this nonrenormalization theorem to the renormalized action discussed in this paper.

These two points indicate that the D-terms, or at least a large subclass of them, may in fact not contribute to the renormalized action.

Adding hyper multiplets does not change the transformation rules of the vector multiplets. We therefore expect that the localizing instantons that we have found here will continue to exist. There could be in principle additional localizing solutions where hyper multiplet fields are excited but this may not necessarily happen. It then only remains to check that the coupling of hyper multiplets and vector multiplets at high order cannot contribute to the renormalized action. Lacking an offshell formulation of couplings between hypers and vectors, we cannot at present address this question but perhaps something analogous to the nonrenormalization theorem discussed above can be extended to these terms as well. 
In any case, these questions can be systematically investigated in the context of our off-shell localizing instantons. If some of the D-terms do happen to contribute to the renormalized action, their contribution can be taken into account by evaluating them on the off-shell solutions. Similarly if there are new localizing instantons upon the inclusion of hypers, those too can be added as separate contribution to the final answer for the functional integral.

We would like to add that in several cases such as small black holes and big black holes in models with $\mathcal{N}=4$ supersymmetry, exact microscopic degeneracies are known [63-68]. One can thus apply the formalism developed here in these specific cases to test the macroscopic results against a known answer from the microscopic side. These applications of our results will be reported in a forthcoming publication [16]. We will only note here that in all these models, the macroscopic answer obtained ignoring D-terms and hyper multiplets appears to agree in remarkable details with the exact microscopic answer. The agreement between microscopic and macroscopic answers thus gives additional evidence that ignoring D-terms and hypermultiplets may be justified at least with $\mathcal{N}=4$ supersymmetry. Unfortunately, a manifestly off-shell formalism is not available for the $\mathcal{N}=4$ theory. So at present, it remains an interesting open problem in offshell supergravity to check to what extent the assumption of ignoring D-terms and hypermultiplets is justified.

If the hyper multiplets and D-terms can be ignored for reasons outlined above, one can conclude that $W_{0}(q, p)$ has the same form as $\hat{W}(q, p)$ evaluated in section 5

$$
W_{0}(q, p)=\int_{\mathcal{M}_{Q}} e^{-\pi \phi^{I} q_{I}}\left|Z_{\mathrm{top}}(\phi, p)\right|^{2} Z_{\operatorname{det}}[d C]_{\mu}
$$

The contribution from the orbifolds of $A d S_{2}$ also has a very similar structure since the localizing instanton solution is still valid.

\subsection{Non-perturbative corrections from orbifolds of $A d S_{2}$}

As we have seen the quantum entropy receives contributions not only from local higherderivative terms in the effective action via the Wald formula but also from nonlocal effects arising from integrating out massless fields. As discussed, upon localization, these nonlocal effects are entirely captured by the ordinary integrals over the parameters $\left\{C^{I}\right\}$ and $Z_{\text {det }}$. The nonperturbative corrections due to brane instantons are included in the integrand by $\left|Z_{\text {inst }}\right|^{2}$.

This is, however, not the whole story. There are additional nonperturbative corrections which arise from subleading gravitational saddle points with the same $A d S_{2}$ boundary conditions but whose action is exponentially suppressed given by the terms with positive $s$ in (1.6). Localization allows us to evaluate the contribution for each family of these subleading saddle points as well, with a structure similar to the leading saddle points. Even though these saddle points are exponentially suppressed, it is meaningful to keep them relative to the power-law suppressed terms, because the evaluation of the functional integral using localization is exact and not an asymptotic expansion.

Based on the structure of known microscopic degeneracy formulas, it was proposed in $[22,69]$ that there is a universal series of saddle points to the path integral semiclassical 
solutions of the theory with the same boundary conditions as the pure $A d S_{2}$, but which differs in the bulk. These saddle points give non-perturbative corrections to the entropy of the form $e^{\mathcal{E}_{0} / c}$ for $c=2,3 \ldots$ These solutions are orbifolds of $A d S_{2} \times M$, where the orbifold action on the $A d S_{2}$ factor of the geometry is a $\mathbb{Z}_{c}$ quotient in the angular direction of Euclidean $A d S_{2}$ :

$$
d s^{\prime 2}=v\left[\left(r^{\prime 2}-1\right) d{\theta^{\prime}}^{2}+\frac{d r^{\prime 2}}{{r^{\prime}}^{2}-1}\right], \quad \theta^{\prime} \sim \theta^{\prime}+2 \pi / c .
$$

so that indeed the bulk action gets reduced by a factor of $c$ compared to the original solution $c=1$. By a change of coordinates $r^{\prime}=c r, \theta^{\prime}=\theta / c$, one gets

$$
d s^{2}=v\left[\left(r^{2}-\frac{1}{c^{2}}\right) d \theta^{2}+\frac{d r^{2}}{r^{2}-\frac{1}{c^{2}}}\right], \quad \theta \sim \theta+2 \pi,
$$

which makes it manifest that the asymptotics of all the solutions are the same as required (2.5).

These solutions of course have a conical singularity at the center of $A d S_{2}$, which we do not a priori expect to be included in the sum over configurations. To remedy this, [69] proposed that only such singularities which can be resolved in string theory should be allowed in this sum. This includes orbifolds which, in addition to the above action, simultaneously act as a rotation on the internal $S^{2}$ which is generically present in four dimensional black holes. In this case, the singularity is locally of the $\mathbb{C}^{2} / Z_{n}$ type which is known to be resolvable in string theory after the addition of a $\mathrm{B}$ field on a collapsing cycle as in the case of usual orbifold singularities.

In [70], it was noticed that there is another series of saddle points associated with each electric charge that the black hole carries. ${ }^{11}$ The construction is as follows - corresponding to each electric charge $q_{i}$, one has a gauge potential $A_{i}$ which classically is of the form (2.3). Along with the above action (6.2), if one simultaneously makes a gauge transformation with a constant parameter (thus generating a constant gauge field at asymptotic infinity), then the orbifold action has no fixed point in the full configuration space which includes the geometry and all the Wilson lines, and therefore there is no singularity. In the Euclidean theory, if the orbifold action respects the supersymmetries, then it must also involve a rotation in the $S^{2}[69]$.

These smooth orbifolds are labelled (for each charge $q_{i}$ ) by a pair of integers $\left(c, d_{i}\right)$ and $c \geq 1$. Changing $d \rightarrow d+c$ does not change the Wilson line, and so one has $1 \leq d_{i}<c$. In addition, one must demand that there are no new fixed points that arise, and this gives the condition that $\left(c,\left\{d_{i}\right\}\right)$ are relatively prime.

In the case that the electric charge $q_{i}$ has an interpretation as a momentum on a compact circle in the internal geometry $M$, then the above construction gets a geometric

\footnotetext{
${ }^{11}$ The second type of orbifold can be called 'electric' in that it can be understood simply as an insertion of a Wilson line associated with an electric charge, along with a geometric action on the $A d S_{2}$. The first type can be called 'magnetic' in that it can be understood in similar terms as the insertion of an 't Hooft line corresponding to a magnetic charge [70].
} 
life in the three dimensional theory as the very near-horizon limit of a family of extremal BTZ black holes with the same mass and charge [70]. In this case, we can think of the inequivalent solutions are labelled by double cosets $\Gamma_{\infty} \backslash \mathrm{SL}(2, \mathbb{Z}) / \Gamma_{\infty}$ where

$$
\Gamma_{\infty}=\left(\begin{array}{ll}
1 & * \\
0 & 1
\end{array}\right)
$$

This family can be thought of as related to a different family of black holes in asymptotically $A d S_{3}$ space with fixed electric potential [71] by a Laplace transform in spacetime. These two families of orbifolds together were sufficient to give a semiclassical interpretation of the microscopic partition function of supersymmetric black holes in $\mathcal{N}=4$ theories.

In the $\mathcal{N}=2$ situation, there are no geometric one-cycles in $M$ in the weak coupling limit (although there is always one $S^{1}$ in the M-theory frame). However, the general construction sketched above applies for each of the electric charges which arise from branes wrapping cycles inside the Calabi-Yau, and each such charge should give rise to a family of saddle points. In general, these families may not all be independent, and may be related by the duality symmetries that preserve a given charge configuration. This is indeed the case in the $\mathcal{N}=4$ theory where we know the microscopic answer - there is only one family of solutions for each T-duality invariant. From the $\mathcal{N}=4$ answer, it seems like the 't Hooft line construction applies to each of the magnetic charges as well (up to T-duality as above), but S-duality does not correlate the electric and magnetic family of solutions, and they have independent labels.

\subsection{Relation to classical entropy function and the topological string}

We would like to conclude with a few comments about the relation of our results with the topological string and earlier works.

- One of the remarkable facts about the formula (6.1) is that the renormalized action that appears in the integrand is precisely the classical entropy function as $\exp [\mathcal{E}(\phi, p)][58]$. As mentioned in the introduction, the classical entropy function $\mathcal{E}$ is simply an elegant way to summarize the classical attractor equations. It is simply a Legendre transform of the classical action with respect to the electric charges, and its value at the critical point equals the Wald entropy. Since the definition of the entropy function is purely classical, only the critical points of this function and the value of the function at the critical point have any physical meaning. This fixes only two terms in the Taylor expansion around the critical point and thus there are any number of functions with the same critical point and the same value at the critical point. It is not clear which will play the role of an off-shell action. Ooguri, Strominger, and Vafa of [21] made an inspired guess to attribute meaning to the entropy function away from critical point and elevated it to an off-shell action of a mixed ensemble. This means that to obtain the degeneracy, one must effectively elevate the classical Legendre transform to an inverse Laplace transform. Our derivation of the localization action explains from first principles why such an off-shell action can make sense. Moreover, it gives a systematic way to determine contributions from one-loop 
determinants and brane-instantons. The integration measure is inherited from the supergravity measure and since the range of $C$ fields is the entire real line the $\phi$ contours of integral are parallel to imaginary axis as for any inverse Laplace transform.

- If we ignore $\left|Z_{\text {inst }}\right|^{2}$ and $Z_{\text {det }}$ in (6.1) then $\left|Z_{\text {top }}(\phi, p)\right|^{2}$ could be regarded precisely as the mixed ensemble partition function of OSV conjecture with the measure for the inverse Laplace transform understood to be determined as above. A derivation of this conjecture has been outlined in [72] which uses $A d S_{3}$ geometry and dilute gas approximation. This is justified in the region where the MSW picture gives the complete result and for range of charges for which dilute gas of M2-branes and anti M2-branes dominates the path integral. By contrast, methods outlined in this paper provide a way to derive the quantum entropy for arbitrary charges without any restriction.

- A derivation of the $\left|Z_{\text {top }}(\phi, p)\right|^{2}$ factor for all orders in perturbation theory in a large charge expansion has been suggested in $[17,73]$ using world sheet string theory in the $A d S_{2}$ background. In this picture worldsheet instantons at the north pole contribute $Z_{\text {top }}$ whereas worldsheet instantons at the south pole contribute $\bar{Z}_{\text {top }}$ giving a new perspective on the form of the integrand. These string worldsheet computations are necessarily tied to a fixed classical background which solves the string equations of motion. As we have seen, the $A d S_{2}$ classical background does not access arbitrary values of the electric potential $\phi$ which are fixed to the attractor value. Our localizing instanton solution provides a way access the large field regions of the functional integral away from the critical point. However perturbative worldsheet calculations would not be applicable around such off-shell configurations and it is necessary to evaluate the supergravity functional integral.

- The $A d S_{2}$ boundary conditions require to hold fixed the charges and the electric fields at the boundary have to held fixed at their attracror value. Moreover, the integration variables are not really the electric fields $e^{I}$ conjugate to the charge $e_{I}$ but rather the parameters $C^{I}$ which is the value of the auxiliary fields at the origin of the $A d S_{2}$. Indeed, integration over the electric field as suggested by the OSV conjecture presented an important conceptual difficulty since it would appear to be in conflict with the usual rules of $A d S_{2}$ holography. Such an integration would imply integrating over different boundary conditions. Our derivation of the integral (5.49) shows that the electric field indeed remains fixed at the attractor values. The integration over the parameters $C$ enters into the story for entirely different reasons having to do with localization. All auxiliary fields for the localizing solutions vanish at the boundary and thus respect the $A d S_{2}$ boundary conditions.

- As we have seen in section 6.2 , in string theory there are additional nonperturbative corrections that arise from gravitational saddle points with the same $A d S_{2}$ boundary conditions. These can be viewed as orbifolds of the leading solution. As a result, their contribution has a very similar structure to (6.1). These contributions also indicate 
$W(q, p)$ in a microcanonical ensemble is a more natural object than the integrand of (5.49) which could be thought of the partition function in a mixed ensemble. Conversely, one can use the knowledge of the microscopic answer to figure out the rules of nonperturbative quantum gravity to determine which configurations have to be included in the functional integral.

- The quantum degeneracies of black holes $d(q, p)$ in an appropriate duality frame are given by the Donaldson-Thomas invariants. Given an exact evaluation of the same quantity from macroscopic side in terms of $W(q, p)$ involves the topological string partition function which is related to the Gromov-Witten invariants. Results in this paper can provide a way to establish a precise relation between these two very different counting problems. Note however that $W(q, p)$ give the degeneracies of a single black hole horizon. To compare with the microscopic side it is necessary to separate the contribution from single-centered black holes to extract from the Donaldson-Thomas invariants which count the quantum states in asymptotically flat spacetime. This is an interesting mathematical problem in itself. In an analogous $\mathcal{N}=4$ situation a complete solution of this problem is known [74]. It may be possible in simple examples to arrive at 'exact holography' where both bulk and boundary partition functions can be computed exactly [16]. This connection could be used conversely to figure out the rules of nonperturbative functional integral of quantum gravity and string field theory in $A d S_{2}$ using the knowledge of the black hole degeneracies since one then knows what the functional integral must evaluate to. Perhaps there is a 'twisted' version of this functional integral of string field theory that will focus directly on this 'topological' BPS sector of the theory.

\section{Acknowledgments}

We would like to thank Chris Beasley, Bernard de Wit, Nadav Drukker, Vasily Pestun, and Amir Keshani-Poor for useful discussions. We are especially grateful to Ashoke Sen for several illuminating discussions throughout the course of this work. The work of A. D. was supported in part by the Excellence Chair of the Agence Nationale de la Recherche (ANR). The work of J. G. is supported in part by Fundacão para Ciência e Tecnologia (FCT). The work of S. M. is supported in part by the European Commission Marie Curie Fellowship under the contract PIIF-GA-2008-220899.

\section{A Killing spinors in the attractor geometry}

To apply localization arguments, it is necessary to identify the supercharge $Q$ that squares to the compact bosonic generator $L-J$. For this purpose, it is useful to know first the explicit form of the Killing spinors in the on-shell attractor geometry.

Recall that in the superconformal formalism, there are fermionic variations corresponding to $Q$ as well as $S$, which we label by $\varepsilon$ and $\eta$ respectively [45]. One can only impose $Q$-invariance up to a uniform $S$-supertranslation. This corresponds to the fact that the 
physical supersymmetries in the Poincaré theory are found after the gauge fixing procedure to be a linear combination of these two variations. In general, this combination has a complicated dependence on the other fields as well as the choice of prepotential. The method of [47] is to surpass this problem by finding spinor fields whose variation under $S$ vanishes. One can then simply use the $Q$-invariance conditions for these spinor fields, which by construction is gauge independent. This construction was very useful in [47] to find the half-BPS solution in asymptotically flat space.

However, these gauge-independent supersymmetry transformations then depend on the choice of prepotential and hence the choice of the Lagrangian. This is not well-suited for our purposes since we are really interested in the off-shell localizing solutions that are determined direcly by the off-shell supersymmetry transformation without any reference to the prepotential. Moreover, we are only interested in the near horizon geometry which is much simpler to analyze than the full black hole solution including the asymptotic infinity. For the near horizon supersymmetries, we make the simple observation that a choice of the bosonic fields corresponding to the near horizon attractor background leads to a particularly simple choice of gauge for the physical theory, namely $\eta=0$. This choice then permits us to work with the simpler supersymmetry transformations of the superconformal theory.

To see this, we begin by imposing the vanishing of the variations of fermionic fields of the Weyl mutiplet:

$$
\begin{aligned}
0 & =\delta \psi_{\mu}^{i}=2 D_{\mu} \epsilon^{i}-\frac{1}{8} \gamma_{a} \gamma_{b} T^{a b i j} \gamma_{\mu} \epsilon_{j}+\gamma_{\mu} \eta^{i} \\
0 & =\delta \chi^{i}=-\frac{1}{12} \gamma_{a} \gamma_{b} \not D T^{a b i j} \epsilon_{j}+D \epsilon^{i}+\frac{1}{12} T_{a b}^{i j} \gamma^{a} \gamma^{b} \eta_{j} \\
0 & =\delta \phi_{\mu}^{i}=-2 f_{\mu}^{a} \gamma_{a} \epsilon^{i}-\frac{1}{4} \not D T_{c d}^{i j} \sigma^{c d}+2 D_{\mu} \eta^{i}
\end{aligned}
$$

At the attractor values, we have

$$
v=\frac{16}{\omega \bar{\omega}}, \quad T_{r t}^{-}=v \omega
$$

and the above variations simplify to

$$
\begin{aligned}
\delta \psi_{\mu}^{i} & =2 D_{\mu} \epsilon^{i}-\frac{1}{8} \gamma_{a} \gamma_{b} T^{a b i j} \gamma_{\mu} \epsilon_{j}+\gamma_{\mu} \eta^{i} \\
\delta \chi^{i} & =\frac{1}{12} T_{a b}^{i j} \gamma^{a} \gamma^{b} \eta_{j} \\
\delta \phi_{\mu}^{i} & =2 D_{\mu} \eta^{i} .
\end{aligned}
$$

From here, we deduce the $A d S_{2} \times S^{2}$ Killing spinors equations

$$
\begin{aligned}
D_{\mu} \epsilon^{i} & =\frac{1}{16} \gamma_{a} \gamma_{b} T^{a b i j} \gamma_{\mu} \epsilon_{j}, \\
D_{\mu} \epsilon_{i} & =\frac{1}{16} \gamma_{a} \gamma_{b} T^{a b}{ }_{i j} \gamma_{\mu} \epsilon^{j} \\
\eta_{i}=\eta^{i} & =0 .
\end{aligned}
$$


We thus see that $\eta^{i}=0$ as promised. Before solving the equation for $\epsilon^{i}, \epsilon_{i}$, note that in the Euclidean theory in four dimensions, the spinors should have a symplectic-Majorana condition imposed on them, while in Minkowski spacetime they can be majorana or symplecticMajorana [75]. In addition, the Weyl projection is not compatible with the majorana condition in the Minkowski case and therefore the left and right-handed spinors are complex conjugate to each other. On the contrary, in the Euclidean case, we can have symplectic Majorana-Weyl spinors but not majorana

$$
\left(\zeta_{ \pm}^{i}\right)^{*}=-i \varepsilon_{i j}\left(\sigma_{1} \times \sigma_{2}\right) \zeta_{ \pm}^{j}
$$

where the indices $i, j$ are $\mathrm{SU}(2)^{\prime}$ quantum numbers and $\varepsilon_{i j}$ is the antisymmetric tensor of $\mathrm{SU}(2)$. In the literature [45] the spinors used obeyed a majorana condition in Minkowski space. They used the convention that positive/negative chirality is correlated with upper/down $\mathrm{SU}(2)^{\prime}$ indice due to complex conjugation. Since the killing spinor equations (A.8) were derived from the Lorentzian theory, we shall use an ansatz which reproduces the killing spinor equations in Euclidean $A d S_{2} \times S^{2}$. The ansatz is the following

$$
\begin{aligned}
\epsilon_{i} & =i \varepsilon_{i j} \xi_{-}^{j} \\
\epsilon^{i} & =\xi_{+}^{i}
\end{aligned}
$$

Note that we explicitly show the chirality of the spinor. We should therefore solve the Killing spinor condition for an unconstrained Dirac spinor $\xi^{i}=\xi_{+}^{i}+\xi_{-}^{i}$, double the space and then impose the above constraint (A.9). We represent the Dirac spinor $\xi$ as a direct product $\xi=\xi_{\mathrm{AdS}_{2}} \otimes \xi_{\mathrm{S}^{2}}$ where $\xi_{\mathrm{AdS}_{2}}$ and $\xi_{\mathrm{S}^{2}}$ are two component spinors, and use the following gamma matrix representation

$$
\gamma_{\theta}=\sqrt{v} \sinh \eta \sigma_{1} \otimes 1, \quad \gamma_{\eta}=\sqrt{v} \sigma_{2} \otimes 1, \quad \gamma_{\phi}=\sqrt{v} \sin \psi \sigma_{3} \otimes \sigma_{1}, \quad \gamma_{\psi}=\sqrt{v} \sigma_{3} \otimes \sigma_{2},
$$

where $v \equiv v_{1}\left(=v_{2}\right)$ is the classical size of the $A d S_{2}$ (and the $S^{2}$ ).

Equations (A.8) simplify to the diagonal form

$$
\begin{aligned}
D_{\mu} \xi_{\mathrm{AdS}_{2}}^{i} & =\frac{\omega}{|\omega|} \frac{i}{2}\left(\sigma_{3} \times 1\right) \gamma_{\mu} \xi_{\mathrm{AdS}_{2}}^{i}, \\
D_{j} \xi_{\mathrm{S}^{2}}^{i} & =\frac{\omega}{|\omega|} \frac{i}{2}\left(\sigma_{3} \times 1\right) \gamma_{j} \xi_{\mathrm{S}^{2}}^{i} .
\end{aligned}
$$

which are easily solved [76]. In the bispinor basis

$$
\begin{aligned}
\xi & =a_{1}\left(\begin{array}{l}
1 \\
0
\end{array}\right) \times\left(\begin{array}{l}
1 \\
0
\end{array}\right)+a_{2}\left(\begin{array}{l}
0 \\
1
\end{array}\right) \times\left(\begin{array}{l}
1 \\
0
\end{array}\right)+a_{3}\left(\begin{array}{l}
1 \\
0
\end{array}\right) \times\left(\begin{array}{l}
0 \\
1
\end{array}\right)+a_{4}\left(\begin{array}{l}
0 \\
1
\end{array}\right) \times\left(\begin{array}{l}
0 \\
1
\end{array}\right) \\
& \equiv\left(\begin{array}{l}
a_{1} \\
a_{2} \\
a_{3} \\
a_{4}
\end{array}\right)
\end{aligned}
$$


the solutions are (this is assuming that $w \in \mathbb{R}^{+}$, and we have fixed a certain normalization for the spinors):

$$
\begin{gathered}
\xi_{--}^{i}=2 e^{-\frac{i}{2}(\theta+\phi)}\left(\begin{array}{c}
\cosh \frac{\eta}{2} \cos \frac{\psi}{2} \\
\sinh \frac{\eta}{2} \cos \frac{\psi}{2} \\
-\cosh \frac{\eta}{2} \sin \frac{\psi}{2} \\
-\sinh \frac{\eta}{2} \sin \frac{\psi}{2}
\end{array}\right), \quad \xi_{-+}^{i}=2 e^{-\frac{i}{2}(\theta-\phi)}\left(\begin{array}{c}
\cosh \frac{\eta}{2} \sin \frac{\psi}{2} \\
\sinh \frac{\eta}{2} \sin \frac{\psi}{2} \\
\cosh \frac{\eta}{2} \cos \frac{\psi}{2} \\
\sinh \frac{\eta}{2} \cos \frac{\psi}{2}
\end{array}\right), \\
\xi_{+-}^{i}=2 e^{\frac{i}{2}(\theta-\phi)}\left(\begin{array}{c}
\sinh \frac{\eta}{2} \cos \frac{\psi}{2} \\
\cosh \frac{\eta}{2} \cos \frac{\psi}{2} \\
-\sinh \frac{\eta}{2} \sin \frac{\psi}{2} \\
-\cosh \frac{\eta}{2} \sin \frac{\psi}{2}
\end{array}\right), \quad \xi_{++}^{i}=2 e^{\frac{i}{2}(\theta+\phi)}\left(\begin{array}{c}
\sinh \frac{\eta}{2} \sin \frac{\psi}{2} \\
\cosh \frac{\eta}{2} \sin \frac{\psi}{2} \\
\sinh \frac{\eta}{2} \cos \frac{\psi}{2} \\
\cosh \frac{\eta}{2} \cos \frac{\psi}{2}
\end{array}\right) .
\end{gathered}
$$

As explained above, we should impose a symplectic-Majorana conditon on the spinors. In the above basis, equation (A.9) implies:

$$
\begin{aligned}
& \xi_{++}^{+}=\left(\xi_{--}^{-}\right)^{*} \\
& \xi_{-+}^{+}=\left(\xi_{+-}^{-}\right)^{*} \\
& \xi_{++}^{-}=\left(-\xi_{--}^{+}\right)^{*} \\
& \xi_{-+}^{-}=\left(-\xi_{+-}^{+}\right)^{*}
\end{aligned}
$$

where the star is not the ordinary complex conjugation but the complex conjugation condition as defined by the symplectic-majorana condition.

One can now identify the spinors $\epsilon_{r a}^{i}$ as the generators of $G_{r}^{i a}$, the supercharges of the near horizon $\mathcal{N}=4$ superalgebra $\S 3.2$. The real combinations $Q_{\mu}, \widetilde{Q}_{\mu}, \mu=1, \ldots, 4$ are generated by the combinations:

$$
\begin{array}{ll}
\zeta_{1}=\xi_{++}^{+}+\xi_{--}^{-}, & \tilde{\zeta}_{1}=\xi_{-+}^{+}+\xi_{+-}^{-}, \\
\zeta_{2}=-i\left(\xi_{++}^{+}-\xi_{--}^{-}\right), & \tilde{\zeta}_{2}=-i\left(\xi_{-+}^{+}-\xi_{+-}^{-}\right), \\
\zeta_{3}=-i\left(\xi_{++}^{-}+\xi_{--}^{+}\right), & \tilde{\zeta}_{3}=-i\left(\xi_{-+}^{-}+\xi_{+-}^{+}\right), \\
\zeta_{4}=\xi_{++}^{-}-\xi_{--}^{+}, & \tilde{\zeta}_{4}=\xi_{-+}^{-}-\xi_{+-}^{+},
\end{array}
$$

We can easily see that these killing spinors are real under the complex conjugation condition defined by (A.9). As an instructive exercise take for example $\zeta^{1}$. The $S U(2)^{\prime}$ components are $\zeta^{1+}=\xi_{++}^{+}$and $\zeta^{1-}=\xi_{--}^{-}$. Both are complex conjugate to each other

$$
\begin{aligned}
& \left(\zeta^{1+}\right)^{*}=-i \varepsilon_{+-}\left(\sigma_{1} \times \sigma_{2}\right) \zeta^{1-} \\
& \left(\zeta^{1-}\right)^{*}=-i \varepsilon_{-+}\left(\sigma_{1} \times \sigma_{2}\right) \zeta^{1+}
\end{aligned}
$$

Recall that the supersymmetry variations for fermions and scalars of the vector multiplets in Minkowski theory are [45]

$$
\begin{aligned}
\delta X^{I} & =\bar{\epsilon}^{i} \Omega_{i}^{I} \\
\delta \bar{X}^{I} & =\bar{\epsilon}_{i} \Omega^{I i} \\
\delta \Omega_{i}^{I} & =2 \not \partial X^{I} \epsilon_{i}+\frac{1}{2} \varepsilon_{i j} \mathcal{F}^{I \mu \nu-} \gamma_{\mu} \gamma_{\nu} \epsilon^{j}+Y_{i j}^{I} \epsilon^{j}+2 X^{I} \eta_{i} \\
\delta \Omega^{I i} & =2 \not \partial \bar{X}^{I} \epsilon^{i}+\frac{1}{2} \varepsilon^{i j} \mathcal{F}^{I \mu \nu+} \gamma_{\mu} \gamma_{\nu} \epsilon_{j}+Y^{I i j} \epsilon_{j}+2 \bar{X}^{I} \eta^{i}
\end{aligned}
$$


where $\Omega_{i}$ has positive chirality while $\Omega^{i}$ has negative chirality. Changing basis from the $\epsilon$ spinors to the $\zeta$ spinors using (A.10), we can reexpress the susy variations as

$$
\begin{aligned}
\delta X^{I} & =-\left(\zeta_{+}^{i}\right)^{\dagger} \lambda_{+}^{I i} \\
\delta \bar{X}^{I} & =-\left(\zeta_{-}^{i}\right)^{\dagger} \lambda_{-}^{I i} \\
\delta \lambda_{+}^{I i} & =\frac{1}{2}\left(F_{\mu \nu}^{I-}-\frac{1}{4} \bar{X}^{I} T_{\mu \nu}^{-}\right) \gamma^{\mu} \gamma^{\nu} \zeta_{+}^{i}+2 i \not \partial X^{I} \zeta_{-}^{i}+Y_{j}^{I i} \zeta_{+}^{j} \\
\delta \lambda_{-}^{I i} & =\frac{1}{2}\left(F_{\mu \nu}^{I+}-\frac{1}{4} X^{I} T_{\mu \nu}^{+}\right) \gamma^{\mu} \gamma^{\nu} \zeta_{-}^{i}+2 i \partial \bar{X}^{I} \zeta_{+}^{i}+Y_{j}^{I i} \zeta_{-}^{j}
\end{aligned}
$$

where $\lambda$ are related to $\Omega$ spinors by

$$
\Omega_{i}=\varepsilon_{i j} \lambda_{-}^{j} \quad \Omega^{i}=-i \lambda_{+}^{i}
$$

Under a transformation generated by $\zeta_{i}$ or $\tilde{\zeta}_{i}$, given in (A.17), we can show that the action of $\delta^{2}$ is $L-J$ or $L+J$ respectively

$$
\begin{aligned}
& \delta^{2} X^{I}=-\left(\zeta_{+}^{i}\right)^{\dagger} \delta \lambda_{+}^{I i}=2 i\left(\zeta_{+}^{i}\right)^{\dagger} \partial X^{I} \zeta_{-}^{i} \\
& \delta^{2} \bar{X}^{I}=-\left(\zeta_{-}^{i}\right)^{\dagger} \delta \lambda_{-}^{I i}=2 i\left(\zeta_{-}^{i}\right)^{\dagger} \partial \bar{X}^{I} \zeta_{+}^{i}
\end{aligned}
$$

where the remaining contractions vanish identically for the spinors chosen. After a straightforward computation we find

$$
\begin{aligned}
& \delta^{2} X^{I}=-2 i\left(\partial_{\theta}-\partial_{\phi}\right) X^{I}=2(L-J) X^{I} \\
& \delta^{2} \bar{X}^{I}=-2 i\left(\partial_{\theta}-\partial_{\phi}\right) \bar{X}^{I}=2(L-J) \bar{X}^{I} .
\end{aligned}
$$

\section{B Some aspects of the superconformal multiplet calculus}

In this appendix, we shall summarize some aspects of the superconformal multiplet calculus which we briefly presented in section 4 . We shall first present the supersymmetry variation of the various multiplets. We shall then present the invariant Lagrangian density formula for a chiral multiplet. We shall then present the rule which defines the various components of a scalar function of chiral superfields e.g. the prepotential superfield $\mathbf{F}\left(\mathbf{X}^{\mathbf{I}}\right)$. These are the basic ingredients that go into building the superconformal action. We shall borrow the presentation of the recent [13] wherein a lot of these facts (and more) have been collected, this can be referred to for more details.

The invariance of the bulk Lagrangian under the superconformal transformations are well established, we provide these details for the sake of completeness. Using the same transformations, in another appendix, we shall sketch the supersymmetry invariance of our conjectured boundary action. This, as far as we know, is new, and there is scope to develop it further. 
As in the text, $\epsilon_{i}$ and $\eta_{i}$ denote the parameters of the $Q$ and $S$ supersymmetry transformations. The transformation rules for a chiral multiplet of Weyl weight $w$ are:

$$
\begin{aligned}
\delta A= & \bar{\epsilon}^{i} \Psi_{i} \\
\delta \Psi_{i}= & 2 \not D A \epsilon_{i}+B_{i j} \epsilon^{j}+\frac{1}{2} \gamma^{a b} F_{a b}^{-} \varepsilon_{i j} \epsilon^{j}+2 w A \eta_{i} \\
\delta B_{i j}= & 2 \bar{\epsilon}_{(i} \not D \Psi_{j)}-2 \bar{\epsilon}^{k} \Lambda_{(i} \varepsilon_{j) k}+2(1-w) \bar{\eta}_{(i} \Psi_{j)}, \\
\delta F_{a b}^{-}= & \frac{1}{2} \varepsilon^{i j} \bar{\epsilon}_{i} \not D \gamma_{a b} \Psi_{j}+\frac{1}{2} \bar{\epsilon}^{i} \gamma_{a b} \Lambda_{i}-\frac{1}{2}(1+w) \varepsilon^{i j} \bar{\eta}_{i} \gamma_{a b} \Psi_{j} \\
\delta \Lambda_{i}= & -\frac{1}{2} \gamma^{a b} \not D F_{a b}^{-} \epsilon_{i}-\not D B_{i j} \varepsilon^{j k} \epsilon_{k}+C \varepsilon_{i j} \epsilon^{j}+\frac{1}{4}\left(\not D A \gamma^{a b} T_{a b i j}+w A \not D \gamma^{a b} T_{a b i j}\right) \varepsilon^{j k} \epsilon_{k} \\
& -3 \gamma_{a} \varepsilon^{j k} \epsilon_{k} \bar{\chi}_{[i} \gamma^{a} \Psi_{j]}-(1+w) B_{i j} \varepsilon^{j k} \eta_{k}+\frac{1}{2}(1-w) \gamma^{a b} F_{a b}^{-} \eta_{i}, \\
\delta C= & -2 \varepsilon^{i j} \bar{\epsilon}_{i} \not D \Lambda_{j}-6 \bar{\epsilon}_{i} \chi_{j} \varepsilon^{i k} \varepsilon^{j l} B_{k l} \\
& -\frac{1}{4} \varepsilon^{i j} \varepsilon^{k l}\left((w-1) \bar{\epsilon}_{i} \gamma^{a b} \not D T_{a b j k} \Psi_{l}+\bar{\epsilon}_{i} \gamma^{a b} T_{a b j k} \not D \Psi_{l}\right)+2 w \varepsilon^{i j} \bar{\eta}_{i} \Lambda_{j} .
\end{aligned}
$$

The independent fields of the Weyl multiplet transform as follows,

$$
\begin{aligned}
\delta e_{\mu}{ }^{a} & =\bar{\epsilon}^{i} \gamma^{a} \psi_{\mu i}+\bar{\epsilon}_{i} \gamma^{a} \psi_{\mu}{ }^{i} \\
\delta \psi_{\mu}{ }^{i} & =2 \mathcal{D}_{\mu} \epsilon^{i}-\frac{1}{8} T_{a b}{ }^{i j} \gamma^{a b} \gamma_{\mu} \epsilon_{j}-\gamma_{\mu} \eta^{i} \\
\delta b_{\mu} & =\frac{1}{2} \bar{\epsilon}^{i} \phi_{\mu i}-\frac{3}{4} \bar{\epsilon}^{i} \gamma_{\mu} \chi_{i}-\frac{1}{2} \bar{\eta}^{i} \psi_{\mu i}+\text { h.c. }+\Lambda_{K}^{a} e_{\mu a}, \\
\delta A_{\mu} & =\frac{1}{2} \mathrm{i} \bar{\epsilon}^{i} \phi_{\mu i}+\frac{3}{4} \mathrm{i} \bar{\epsilon}^{i} \gamma_{\mu} \chi_{i}+\frac{1}{2} \mathrm{i} \bar{\eta}^{i} \psi_{\mu i}+\text { h.c. } \\
\delta \mathcal{V}_{\mu}{ }^{i}{ }_{j} & =2 \bar{\epsilon}_{j} \phi_{\mu}{ }^{i}-3 \bar{\epsilon}_{j} \gamma_{\mu} \chi^{i}+2 \bar{\eta}_{j} \psi_{\mu}{ }^{i}-(\text { h.c. } ; \text { traceless }), \\
\delta T_{a b}{ }^{i j} & =8 \bar{\epsilon}^{i} R(Q)_{a b}{ }^{j]} \\
\delta \chi^{i} & =-\frac{1}{12} \gamma^{a b} \not D T_{a b}{ }^{i j} \epsilon_{j}+\frac{1}{6} R(\mathcal{V})_{\mu \nu}{ }^{i}{ }_{j} \gamma^{\mu \nu} \epsilon^{j}-\frac{1}{3} \mathrm{i} R_{\mu \nu}(A) \gamma^{\mu \nu} \epsilon^{i}+D \epsilon^{i}+\frac{1}{12} \gamma_{a b} T^{a b i j} \eta_{j}, \\
\delta D & =\bar{\epsilon}^{i} \not D \chi_{i}+\bar{\epsilon}_{i} \not D \chi^{i}
\end{aligned}
$$

where

$$
R(Q)_{\mu \nu}^{i}=2 \mathcal{D}_{[\mu} \psi_{\nu]}^{i}-\gamma_{[\mu} \phi_{\nu]}^{i}-\frac{1}{8} T^{a b i j} \gamma_{a b} \gamma_{[\mu} \psi_{\nu] j}
$$

Based on these two multiplets, one can write down a Lagrangian density for the chiral multiplet which is invariant under the superconformal transformations:

$$
\begin{aligned}
e^{-1} \mathcal{L}= & C-\varepsilon^{i j} \bar{\psi}_{\mu i} \gamma^{\mu} \Lambda_{j}-\frac{1}{8} \bar{\psi}_{\mu i} T_{a b j k} \gamma^{a b} \gamma^{\mu} \Psi_{l} \varepsilon^{i j} \varepsilon^{k l}-\frac{1}{16} A\left(T_{a b i j} \varepsilon^{i j}\right)^{2} \\
& -\frac{1}{2} \bar{\psi}_{\mu i} \gamma^{\mu \nu} \psi_{\nu j} B_{k l} \varepsilon^{i k} \varepsilon^{j l}+\varepsilon^{i j} \bar{\psi}_{\mu i} \psi_{\nu j}\left(F^{-\mu \nu}-\frac{1}{2} A T_{k l}^{\mu \nu} \varepsilon^{k l}\right) \\
& -\frac{1}{2} \varepsilon^{i j} \varepsilon^{k l} e^{-1} \varepsilon^{\mu \nu \rho \sigma} \bar{\psi}_{\mu i} \psi_{\nu j}\left(\bar{\psi}_{\rho k} \gamma_{\sigma} \Psi_{l}+\bar{\psi}_{\rho k} \psi_{\sigma j} A\right) .
\end{aligned}
$$

This density is built such that the variation of the Lagrangian is equal to a total derivative in spacetime.

The Lagrangian for vector multiplets is based on first viewing the gauge invariant quantities of the vector multiplet as a reduced chiral multiplet with weight $w=1$. The 
components are:

$$
\begin{aligned}
\left.A\right|_{\text {vector }}= & X \\
\left.\Psi_{i}\right|_{\text {vector }}= & \Omega_{i}, \\
\left.B_{i j}\right|_{\text {vector }}= & Y_{i j}=\varepsilon_{i k} \varepsilon_{j l} Y^{k l}, \\
\left.F_{a b}^{-}\right|_{\text {vector }}= & \left(\delta_{a b}{ }^{c d}-\frac{1}{2} \varepsilon_{a b}{ }^{c d}\right) e_{c}{ }^{\mu} e_{d}^{\nu} \partial_{[\mu} A_{\nu]} \\
& +\frac{1}{4}\left[\bar{\psi}_{\rho}^{i} \gamma_{a b} \gamma^{\rho} \Omega^{j}+\bar{X} \bar{\psi}_{\rho}^{i} \gamma^{\rho \sigma} \gamma_{a b} \psi_{\sigma}^{j}-\bar{X} T_{a b}{ }^{i j}\right] \varepsilon_{i j}, \\
\left.\Lambda_{i}\right|_{\text {vector }}= & -\varepsilon_{i j} \not D \Omega^{j}, \\
\left.C\right|_{\text {vector }}= & -2 \square_{\mathrm{c}} \bar{X}-\frac{1}{4} F_{a b}^{+} T^{a b}{ }_{i j} \varepsilon^{i j}-3 \bar{\chi}_{i} \Omega^{i} .
\end{aligned}
$$

The transformations of the vector multiplet are:

$$
\begin{aligned}
\delta X & =\bar{\epsilon}^{i} \Omega_{i}, \\
\delta \Omega_{i} & =2 \not D X \epsilon_{i}+\frac{1}{2} \varepsilon_{i j} F_{\mu \nu} \gamma^{\mu \nu} \epsilon^{j}+Y_{i j} \epsilon^{j}+2 X \eta_{i}, \\
\delta A_{\mu} & =\varepsilon^{i j} \bar{\epsilon}_{i}\left(\gamma_{\mu} \Omega_{j}+2 \psi_{\mu j} X\right)+\varepsilon_{i j} \bar{\epsilon}^{i}\left(\gamma_{\mu} \Omega^{j}+2 \psi_{\mu}{ }^{j}\right), \\
\delta Y_{i j} & =2 \bar{\epsilon}_{(i} \not D \Omega_{j)}+2 \varepsilon_{i k} \varepsilon_{j l} \bar{\epsilon}^{(k} \not D \Omega^{l)} .
\end{aligned}
$$

One then has to choose a meromorphic homogeneous function $F$ of weight 2 and build the multiplet $\mathbf{F}\left(\mathbf{X}^{\mathbf{I}}\right)$ with lowest component $F\left(X^{I}\right)$. The components of this is given in terms of the components of the vector multiplet as follows:

$$
\begin{aligned}
\left.A\right|_{F}= & F(A), \\
\left.\Psi_{i}\right|_{F}= & F(A)_{I} \Psi_{i}{ }^{I}, \\
\left.B_{i j}\right|_{F}= & F(A)_{I} B_{i j}{ }^{I}-\frac{1}{2} F(A)_{I J} \bar{\Psi}_{(i}^{I} \Psi_{j)}{ }^{J}, \\
\left.F_{a b}^{-}\right|_{F}= & F(A)_{I} F_{a b}^{-I}-\frac{1}{8} F(A)_{I J} \varepsilon^{i j} \bar{\Psi}_{i}^{I} \gamma_{a b} \Psi_{j}{ }^{J}, \\
\left.\Lambda_{i}\right|_{F}= & F(A)_{I} \Lambda_{i}{ }^{I}-\frac{1}{2} F(A)_{I J}\left[B_{i j}{ }^{I} \varepsilon^{j k} \Psi_{k}{ }^{J}+\frac{1}{2} F_{a b}^{-I} \gamma^{a b} \Psi_{k}{ }^{J}\right] \\
& +\frac{1}{48} F(A)_{I J K} \gamma^{a b} \Psi_{i}{ }^{I} \varepsilon^{j k} \bar{\Psi}_{j}^{J} \gamma_{a b} \Psi_{k}{ }^{K}, \\
\left.C\right|_{F}= & F(A)_{I} C^{I}-\frac{1}{4} F(A)_{I J}\left[B_{i j}{ }^{I} B_{k l}{ }^{J} \varepsilon^{i k} \varepsilon^{j l}-2 F_{a b}^{-I} F^{-a b J}+4 \varepsilon^{i k} \bar{\Lambda}_{i}{ }^{I} \Psi_{j}{ }^{J}\right], \\
& +\frac{1}{4} F(A)_{I J K}\left[\varepsilon^{i k} \varepsilon^{j l} B_{i j}{ }^{I} \Psi_{k}{ }^{J} \Psi_{l}{ }^{K}-\frac{1}{2} \varepsilon^{k l} \bar{\Psi}_{k}{ }^{I} F_{a b}^{-J} \gamma^{a b} \Psi_{l}{ }^{K}\right] \\
& +\frac{1}{192} F(A)_{I J K L} \varepsilon^{i j} \bar{\Psi}_{i}^{I} \gamma_{a b} \Psi_{j}{ }^{k l} \varepsilon^{k l} \bar{\Psi}_{k}^{K} \gamma_{a b} \Psi_{l}{ }^{L} .
\end{aligned}
$$

\section{Boundary terms and supersymmetry of the renormalized action}

In section 5.3, we conjectured the boundary action (5.43)

$$
\mathcal{S}_{\mathrm{bdry}}=-2 \pi r_{0}\left(\frac{q_{I} e_{*}^{I}}{2}+i\left(F\left(X_{*}^{I}\right)-\bar{F}\left(X_{*}^{I}\right)\right)\right) .
$$

so that $\mathcal{S}_{\text {ren }}$ is finite. We also mentioned that this action is supersymmetric. In this appendix, we shall discuss the action $S_{\text {ren }}$, and show that it is supersymmetric. 
To motivate this, we note that we can rewrite $\mathcal{S}_{\text {ren }}$ as the sum of two pieces

$$
\begin{aligned}
\mathcal{S}_{\text {ren }} & =\mathcal{S}_{\text {bulk }}+\mathcal{S}_{\text {bdry }}+i \frac{q}{2} \oint A \\
& =\left(\mathcal{S}_{\text {bulk }}+\mathcal{S}_{\text {bdry }}^{1}\right)+\left(\frac{i}{2} q_{I} \int_{0}^{2 \pi} A_{\theta}^{I} d \theta+\mathcal{S}_{\text {bdry }}^{2}\right),
\end{aligned}
$$

where we have split the boundary action (5.43) into a sum of two pieces:

$$
\begin{aligned}
& \mathcal{S}_{\text {bdry }}=\mathcal{S}_{\text {bdry }}^{1}+\mathcal{S}_{\text {bdry }}^{2}, \\
& \mathcal{S}_{\text {bdry }}^{1}=-\int_{0}^{2 \pi} i[F(X)-\overline{F(X)}]_{\text {bdry }} e_{\theta}^{\theta} d \theta, \\
& \mathcal{S}_{\text {bdry }}^{2}=-\int_{0}^{2 \pi} \frac{q_{I}}{2}\left[X^{I}+\bar{X}^{I}\right]_{\text {bdry }} e_{\theta}^{\theta} d \theta .
\end{aligned}
$$

Here, $e^{\hat{\theta}}=\sinh \eta_{0}$ is the induced vielbein on the boundary. To verfiy (C.3), we use the same algebra used in (5.40), namely, an expansion of the field $X^{I}$ into its fixed part $X_{*}^{I}$ and varying part which is $\mathcal{O}\left(1 / r_{0}\right)$, followed by a Taylor expansion and the use of attractor equations.

With such a split of the action, the two pieces in (C.2) have a very natural interpretation as we discuss below. We will show further that each of them is finite and supersymmetric, implying the same for $\mathcal{S}_{\text {ren }}$.

Recall that the bulk action (5.37) evaluated on the solution can be written as the difference of two pieces

$$
\mathcal{S}_{\text {bulk }}=2 \pi i r_{0}\left[F\left(X^{I}\right)-\overline{F\left(X^{I}\right)}\right]_{\mathrm{bdry}}-2 \pi i\left[F\left(X^{I}\right)-\overline{F\left(X^{I}\right)}\right]_{\text {origin }} .
$$

We see that $\mathcal{S}_{\text {bulk }}+\mathcal{S}_{\text {bdry }}^{1}$ is manifestly finite. Thus, $\mathcal{S}_{\text {bdry }}^{1}$ has the natural interpretation of a canonical boundary term which cancels the boundary part of the bulk action, so that any variation of $\mathcal{S}_{\text {bulk }}+\mathcal{S}_{\text {bdry }}^{1}$ will be finite and not contain boundary terms.

The second piece of the boundary action combines with the Wilson line to give the operator

$$
\exp \left[-\frac{i}{2} q_{I} \int_{0}^{2 \pi} A_{\theta}^{I} d \theta-\mathcal{S}_{\text {bdry }}^{2}\right]=\exp \left[-\frac{i}{2} q_{I} \int_{0}^{2 \pi}\left(A_{\theta}^{I}+i e_{\theta}^{\theta}\left(X^{I}+\bar{X}^{I}\right)\right) d \theta\right]
$$

This operator has the natural interpretation as the supersymmetric Wilson line of gauge theory $[59,60]$. Recalling the boundary behavior of the fields

$$
\begin{aligned}
-\frac{i}{2} q_{I} \int_{0}^{2 \pi} A_{\theta}^{I} d \theta & =-\pi q_{I} e_{*}^{I} r_{0}\left(1+\mathcal{O}\left(1 / r_{0}\right)\right), \\
-\frac{i}{2} q_{I} \int_{0}^{2 \pi} i e_{\theta}^{\theta}\left(X^{I}+\bar{X}^{I}\right) d \theta & =\pi q_{I} r_{0}\left(X_{*}^{I}+\bar{X}_{*}^{I}+\mathcal{O}\left(1 / r_{0}\right)\right), \\
& =\pi q_{I} e_{*}^{I} r_{0}\left(1+\mathcal{O}\left(1 / r_{0}\right)\right),
\end{aligned}
$$

it is easy to see that this operator is manifestly finite. 
Evaluated on the solutions $A_{\theta}^{I}=-i e_{*}^{I}\left(r_{0}-1\right), X^{I}=X_{*}^{I}+\frac{C^{I}}{r_{0}}, \bar{X}^{I}=\bar{X}_{*}^{I}+\frac{C^{I}}{r_{0}}$ that we consider in section 5 , we see that the two pieces of the renormalized action (C.2) above give the two pieces of the final renormalized action (5.44) which we found in section 5.3, as indeed should happen.

In the rest of the appendix, we shall sketch the proof of supersymmetry of these two operators. The supersymmetry of the operator (C.7) above follows from the transformation rules of $X^{I}$ and $A_{\mu}^{I}$ of the vector multiplet (B.6). We use the fact that the Killing spinors obey

$$
\zeta^{i}=\varepsilon_{i j} \gamma^{0} \zeta^{j}
$$

The extra term in the variation of the vector field which is proportional to the gravitino is cancelled by the variation of the vielbein in the definition of the super Wilson line. This is the new ingredient in the super Wilson line of a gravitational theory compared to that of gauge theory.

Now we come to the supersymmetry of the combination $\mathcal{S}_{\text {bulk }}+\mathcal{S}_{\text {bdry }}^{1}$. The statement that $\mathcal{S}_{\text {bulk }}$ is supersymmetric [10-12] really means that the variation of $\mathcal{S}_{\text {bulk }}$ is a boundary term which can be ignored in certain circumstances. In our situation, there is a non-trivial boundary, and therefore what we need to check is that the variation of the bulk Lagrangian is indeed equal to the derivative of the boundary Lagrangian.

To investigate this, we need to understand the structure of the Lagrangian built using the chiral superfield [48]. In the case of rigid supersymmetry, the variation of the top component of the chiral superfield is a total derivative in spacetime, and therefore the top component (picked by a chiral superspace integral) is an invariant Lagrangian. For chiral superfields coupled to superconformal gravity, the transformation rules undergo a modification and the derivatives become covariant derivatives, and there are additional terms in the variation (B.1) of the top component $C$. The invariant Lagrangian density (B.4) contains new terms whose variation cancel the additional non-derivative terms present in $\delta C$.

The net result of this procedure is that the variation of the invariant Lagrangian is equal to the total derivative terms that are present in the variation of the top component $C$ of the chiral multiplet, i.e. essentially one can drop the extra terms which arise due to the covariantization. As an example, the term proportional to the auxiliary field $B_{i j}$ in $\delta C$ contains $\chi_{i}$ which is an auxiliary field of the superconformal multiplet constrained to be proportional to $R(Q)^{i}$. This term is cancelled by the term proportional to $B_{i j}$ in the higher corrections to the Lagrangian density (B.4) after solving for the auxiliary field $\chi$ in terms of the gravitini.

Looking at the $Q$ variation (B.1) of a chiral multiplet of weight $w=2$, we see that the variation of $C$ contains two total derivative pieces

$$
-2 \varepsilon^{i j} \not \partial\left(\bar{\epsilon}_{i} \Lambda_{j}\right)
$$

and

$$
-\frac{1}{4} \varepsilon^{i j} \varepsilon^{k \ell}\left(\left(\not \partial \bar{\epsilon}_{i} \gamma^{a b} T_{a b j k}\right) \Psi_{l}+\gamma^{a b} T_{a b j k} \not \partial\left(\bar{\epsilon}_{i} \Psi_{\ell}\right)\right)=-\frac{1}{4} \varepsilon^{i j} \varepsilon^{k \ell} \not \partial\left(\bar{\epsilon}_{i} \gamma^{a b} T_{a b j k} \Psi_{\ell}\right) .
$$


In our problem where we have a bunch of vector multiplets, the way to build a Lagrangian is by using the homogeneous function $F\left(X^{I}\right)$. One first builds a chiral multiplet $\mathbf{F}\left(\mathbf{X}^{\mathbf{I}}\right)$ whose bottom component is $F\left(X^{I}\right)$, and then uses the invariant Lagrangian described above for this chiral multiplet. The variation of our Lagrangian is therefore equal to the total derivative terms that appear in the variation of the top component of the chiral multiplet $\mathbf{F}\left(\mathbf{X}^{\mathbf{I}}\right)$. Looking at the form of the components of this superfield (B.7), and then substituting for the components of the reduced chiral multiplet (B.5), we find that the first type of total derivative term from integration of (C.12) is

$$
\begin{aligned}
-\left.2 \varepsilon^{j i} \int_{\text {bulk }} \not \partial\left(\bar{\epsilon}_{j} \Lambda_{i}\right)\right|_{F} \\
=-2 \varepsilon^{j i} \int_{\text {bulk }} \not \partial\left(-\bar{\epsilon}_{j} F(X)_{I} \varepsilon_{i k} \not D \Omega^{k I}-\frac{1}{2} \bar{\epsilon}_{j} F(X)_{I J}\left[B_{i j}{ }^{I} \varepsilon^{j k} \Omega_{k}{ }^{J}+\frac{1}{2} \bar{\epsilon}_{j} F_{a b}^{-I} \gamma^{a b} \Omega_{k}{ }^{J}\right]\right. \\
\left.\quad+\frac{1}{48} \bar{\epsilon}_{j} F(X)_{I J K} \gamma^{a b} \Omega_{i}{ }^{I} \varepsilon^{k \ell} \bar{\Omega}_{k}{ }^{J} \gamma_{a b} \Omega_{\ell}{ }^{K}\right) .
\end{aligned}
$$

We are interested in the bosonic part of the boundary counterterm Lagrangian. The third term on the right hand side contains three fermions and so cannot appear from the variation of a pure bosonic term, so we shall ignore that term here. The second term on the r.h.s. proportional to $F_{I J}$ is equal to the variation of $F_{I J} \Omega^{I} \Omega^{J}$ minus a total derivative term on the boundary. We can therefore drop this term since it is fermionic. Using the variation $\delta X^{I}=\bar{\epsilon}^{i} \Omega_{i}^{I}$, the first term on the r.h.s. is proportional to the variation of the derivative of $F_{I}$, which integrates to zero on the closed boundary, and therefore does not produce any boundary counterterms.

This leaves us with the second term (C.13) which gives rise to a boundary term

$$
-\frac{1}{4} \varepsilon^{i j} \varepsilon^{k \ell} \int_{\mathrm{bulk}} \not \partial\left(\left.\bar{\epsilon}_{i} \gamma^{a b} T_{a b j k} \Psi_{\ell}\right|_{F}\right)=-\frac{1}{4} \varepsilon^{i j} \varepsilon^{k \ell} \int_{\mathrm{bulk}} \not \partial\left(\bar{\epsilon}_{i} \gamma^{a b} T_{a b j k} F(X)_{I} \Omega_{\ell}^{I}\right) .
$$

Now, the variation of $T_{a b j k}$ (B.2) is proportional to the curvature $R(Q)_{a b}$ which integrates to zero on the boundary. Therefore, $T_{a b j k}$ can be treated as a constant on the boundary for the purpose of supersymmetry variations. Plugging in the attractor value for $T_{a b j k}$, and using $\delta X^{I}=\bar{\epsilon}^{i} \Omega_{i}^{I}$ again, and the Killing spinor relation (C.11), we see that the remaining term (C.15) is equal and opposite to the variation of the boundary term $\mathcal{S}_{\text {bdry }}^{1}$, thus showing that the supersymmetry variation of $\mathcal{S}_{\text {bulk }}+\mathcal{S}_{\text {bdry }}^{1}$ vanishes.

Open Access. This article is distributed under the terms of the Creative Commons Attribution Noncommercial License which permits any noncommercial use, distribution, and reproduction in any medium, provided the original author(s) and source are credited.

\section{References}

[1] J.D. Bekenstein, Black holes and entropy, Phys. Rev. D 7 (1973) 2333 [SPIRES].

[2] S.W. Hawking, Particle Creation by Black Holes, Commun. Math. Phys. 43 (1975) 199 [SPIRES].

[3] A. Strominger and C. Vafa, Microscopic Origin of the Bekenstein-Hawking Entropy, Phys. Lett. B 379 (1996) 99 [hep-th/9601029] [SPIRES]. 
[4] A. Sen, Entropy Function and $A d S_{2} / C F T_{1}$ Correspondence, JHEP 11 (2008) 075 [arXiv:0805.0095] [SPIRES].

[5] A. Sen, Quantum Entropy Function from $A d S_{2} / C F T_{1}$ Correspondence, Int. J. Mod. Phys. A 24 (2009) 4225 [arXiv:0809.3304] [SPIRES].

[6] R.M. Wald, Black hole entropy is the Noether charge, Phys. Rev. D 48 (1993) 3427 [gr-qc/9307038] [SPIRES].

[7] V. Iyer and R.M. Wald, Some properties of Noether charge and a proposal for dynamical black hole entropy, Phys. Rev. D 50 (1994) 846 [gr-qc/9403028] [SPIRES].

[8] T. Jacobson, G. Kang and R.C. Myers, Black hole entropy in higher curvature gravity, gr-qc/9502009 [SPIRES].

[9] N. Banerjee, S. Banerjee, R.K. Gupta, I. Mandal and A. Sen, Supersymmetry, Localization and Quantum Entropy Function, JHEP 02 (2010) 091 [arXiv:0905.2686] [SPIRES].

[10] B. de Wit, J.W. van Holten and A. Van Proeyen, Transformation Rules of $N=2$ Supergravity Multiplets, Nucl. Phys. B 167 (1980) 186 [SPIRES].

[11] B. de Wit, P.G. Lauwers and A. Van Proeyen, Lagrangians of $N=2$ Supergravity-Matter Systems, Nucl. Phys. B 255 (1985) 569 [SPIRES].

[12] B. de Wit, J.W. van Holten and A. Van Proeyen, Structure of $N=2$ Supergravity, Nucl. Phys. B 184 (1981) 77 [SPIRES].

[13] B. de Wit, S. Katmadas and M. van Zalk, New supersymmetric higher-derivative couplings: Full $N=2$ superspace does not count!, JHEP 01 (2011) 007 [arXiv:1010.2150] [SPIRES].

[14] V. Pestun, Localization of gauge theory on a four-sphere and supersymmetric Wilson loops, arXiv:0712.2824 [SPIRES].

[15] S. Banerjee, R.K. Gupta and A. Sen, Logarithmic Corrections to Extremal Black Hole Entropy from Quantum Entropy Function, JHEP 03 (2011) 147 [arXiv:1005.3044] [SPIRES].

[16] A. Dabholkar, J. Gomes and S. Murthy, Localization and Exact Holography, in preparation.

[17] C. Beasley et al., Why $Z_{\mathrm{BH}}=\left|Z_{\mathrm{top}}\right|^{2}$, hep-th/0608021 [SPIRES].

[18] N. Banerjee, I. Mandal and A. Sen, Black Hole Hair Removal, JHEP 07 (2009) 091 [arXiv: 0901.0359] [SPIRES].

[19] A. Sen, Two Charge System Revisited: Small Black Holes or Horizonless Solutions?, JHEP 05 (2010) 097 [arXiv: 0908.3402] [SPIRES].

[20] I.R. Klebanov and E. Witten, AdS/CFT correspondence and symmetry breaking, Nucl. Phys. B 556 (1999) 89 [hep-th/9905104] [SPIRES].

[21] H. Ooguri, A. Strominger and C. Vafa, Black hole attractors and the topological string, Phys. Rev. D 70 (2004) 106007 [hep-th/0405146] [SPIRES].

[22] A. Sen, Arithmetic of Quantum Entropy Function, JHEP 08 (2009) 068 [arXiv:0903.1477] [SPIRES].

[23] A. Dabholkar, J. Gomes, S. Murthy and A. Sen, Supersymmetric Index from Black Hole Entropy, JHEP 04 (2011) 034 [arXiv:1009.3226] [SPIRES].

[24] A. Dabholkar, F. Denef, G.W. Moore and B. Pioline, Exact and Asymptotic Degeneracies of Small Black Holes, JHEP 08 (2005) 021 [hep-th/0502157] [SPIRES]. 
[25] A. Dabholkar, F. Denef, G.W. Moore and B. Pioline, Precision counting of small black holes, JHEP 10 (2005) 096 [hep-th/0507014] [SPIRES].

[26] D. Shih and X. Yin, Exact Black Hole Degeneracies and the Topological String, JHEP 04 (2006) 034 [hep-th/0508174] [SPIRES].

[27] G. Lopes Cardoso, B. de Wit, J. Kappeli and T. Mohaupt, Black hole partition functions and duality, JHEP 03 (2006) 074 [hep-th/0601108] [SPIRES].

[28] F. Denef and G.W. Moore, Split states, entropy enigmas, holes and halos, hep-th/0702146 [SPIRES].

[29] G.L. Cardoso, J.R. David, B. de Wit and S. Mahapatra, The mixed black hole partition function for the STU model, JHEP 12 (2008) 086 [arXiv:0810.1233] [SPIRES].

[30] M. Bershadsky, S. Cecotti, H. Ooguri and C. Vafa, Holomorphic anomalies in topological field theories, Nucl. Phys. B 405 (1993) 279 [hep-th/9302103] [SPIRES].

[31] M. Bershadsky, S. Cecotti, H. Ooguri and C. Vafa, Kodaira-Spencer theory of gravity and exact results for quantum string amplitudes, Commun. Math. Phys. 165 (1994) 311 [hep-th/9309140] [SPIRES].

[32] I. Antoniadis, E. Gava, K.S. Narain and T.R. Taylor, Topological amplitudes in string theory, Nucl. Phys. B 413 (1994) 162 [hep-th/9307158] [SPIRES].

[33] R. Jackiw, Liouville Field Theory: a Two-Dimensional Model for Gravity?, MIT-CTP-1049 [SPIRES].

[34] R. Jackiw, Lower Dimensional Gravity, Nucl. Phys. B 252 (1985) 343 [SPIRES].

[35] C. Teitelboim, The Hamiltonian Structure of Two-Dimensional Space-Time and its Relation with the Conformal Anomaly, Print-83-0130 (TEXAS) [SPIRES].

[36] M. Spradlin and A. Strominger, Vacuum states for AdS 2 black holes, JHEP 11 (1999) 021 [hep-th/9904143] [SPIRES].

[37] S.W. Hawking, G.T. Horowitz and S.F. Ross, Entropy, Area and black hole pairs, Phys. Rev. D 51 (1995) 4302 [gr-qc/9409013] [SPIRES].

[38] A. Castro, D. Grumiller, F. Larsen and R. McNees, Holographic Description of AdS $S_{2}$ Black Holes, JHEP 11 (2008) 052 [arXiv: 0809.4264] [SPIRES].

[39] J.J. Duistermaat and G.J. Heckman, On the variation in the cohomology of the symplectic form of the reduced phase space, Invent. Math. 69 (1982) 259.

[40] E. Witten, Topological Quantum Field Theory, Commun. Math. Phys. 117 (1988) 353 [SPIRES].

[41] E. Witten, The $N$ matrix model and gauged WZW models, Nucl. Phys. B 371 (1992) 191 [SPIRES].

[42] E. Witten, Mirror manifolds and topological field theory, hep-th/9112056 [SPIRES].

[43] A.S. Schwarz and O. Zaboronsky, Supersymmetry and localization, Commun. Math. Phys. 183 (1997) 463 [hep-th/9511112] [SPIRES].

[44] O.V. Zaboronsky, Dimensional reduction in supersymmetric field theories, hep-th/9611157 [SPIRES].

[45] T. Mohaupt, Black hole entropy, special geometry and strings, Fortsch. Phys. 49 (2001) 3 [hep-th/0007195] [SPIRES]. 
[46] B. Sahoo and A. Sen, Higher derivative corrections to non-supersymmetric extremal black holes in $N=2$ supergravity, JHEP 09 (2006) 029 [hep-th/0603149] [SPIRES].

[47] G. Lopes Cardoso, B. de Wit, J. Kappeli and T. Mohaupt, Stationary BPS solutions in $N=2$ supergravity with $R^{2}$ interactions, JHEP 12 (2000) 019 [hep-th/0009234] [SPIRES].

[48] M. de Roo, J.W. van Holten, B. de Wit and A. Van Proeyen, Chiral Superfields In $N=2$ Supergravity, Nucl. Phys. B 173 (1980) 175 [SPIRES].

[49] G. Lopes Cardoso, B. de Wit and T. Mohaupt, Corrections to macroscopic supersymmetric black-hole entropy, Phys. Lett. B 451 (1999) 309 [hep-th/9812082] [SPIRES].

[50] G. Lopes Cardoso, B. de Wit and T. Mohaupt, Deviations from the area law for supersymmetric black holes, Fortsch. Phys. 48 (2000) 49 [hep-th/9904005] [SPIRES].

[51] G. Lopes Cardoso, B. de Wit and T. Mohaupt, Area law corrections from state counting and supergravity, Class. Quant. Grav. 17 (2000) 1007 [hep-th/9910179] [SPIRES].

[52] S. Ferrara and R. Kallosh, Supersymmetry and Attractors, Phys. Rev. D 54 (1996) 1514 [hep-th/9602136] [SPIRES].

[53] S. Ferrara, R. Kallosh and A. Strominger, $N=2$ extremal black holes, Phys. Rev. D 52 (1995) 5412 [hep-th/9508072] [SPIRES].

[54] A. Strominger, Macroscopic Entropy of $N=2$ Extremal Black Holes, Phys. Lett. B 383 (1996) 39 [hep-th/9602111] [SPIRES].

[55] G.L. Cardoso, B. de Wit and S. Mahapatra, Black hole entropy functions and attractor equations, JHEP 03 (2007) 085 [hep-th/0612225] [SPIRES].

[56] A. Kapustin, Wilson-'t Hooft operators in four-dimensional gauge theories and S-duality, Phys. Rev. D 74 (2006) 025005 [hep-th/0501015] [SPIRES].

[57] J. Gomis, T. Okuda and D. Trancanelli, Quantum 't Hooft operators and S-duality in $N=4$ super Yang-Mills, Adv. Theor. Math. Phys. 13 (2009) 1941 [arXiv:0904.4486] [SPIRES].

[58] A. Sen, Black Hole Entropy Function, Attractors and Precision Counting of Microstates, Gen. Rel. Grav. 40 (2008) 2249 [arXiv:0708.1270] [SPIRES].

[59] J.M. Maldacena, Wilson loops in large-N field theories, Phys. Rev. Lett. 80 (1998) 4859 [hep-th/9803002] [SPIRES].

[60] S.-J. Rey and J.-T. Yee, Macroscopic strings as heavy quarks in large- $N$ gauge theory and anti-de Sitter supergravity, Eur. Phys. J. C 22 (2001) 379 [hep-th/9803001] [SPIRES].

[61] G.L. Cardoso, B. de Wit and S. Mahapatra, Subleading and non-holomorphic corrections to $N=2$ BPS black hole entropy, JHEP 02 (2009) 006 [arXiv:0808.2627] [SPIRES].

[62] N.A. Nekrasov, Seiberg-Witten Prepotential From Instanton Counting, Adv. Theor. Math. Phys. 7 (2004) 831 [hep-th/0206161] [SPIRES].

[63] R. Dijkgraaf, E.P. Verlinde and H.L. Verlinde, Counting Dyons in $N=4$ String Theory, Nucl. Phys. B 484 (1997) 543 [hep-th/9607026] [SPIRES].

[64] D. Gaiotto, A. Strominger and X. Yin, New Connections Between $4 D$ and 5D Black Holes, JHEP 02 (2006) 024 [hep-th/0503217] [SPIRES].

[65] J.R. David and A. Sen, CHL dyons and statistical entropy function from D1 - D5 system, JHEP 11 (2006) 072 [hep-th/0605210] [SPIRES]. 
[66] A. Dabholkar, J. Gomes and S. Murthy, Counting all dyons in $N=4$ string theory, arXiv:0803.2692 [SPIRES].

[67] A. Dabholkar and J.A. Harvey, Nonrenormalization of the Superstring Tension, Phys. Rev. Lett. 63 (1989) 478 [SPIRES].

[68] A. Dabholkar, G.W. Gibbons, J.A. Harvey and F. Ruiz Ruiz, Superstrings and solitons, Nucl. Phys. B 340 (1990) 33 [SPIRES].

[69] N. Banerjee, D.P. Jatkar and A. Sen, Asymptotic Expansion of the $N=4$ Dyon Degeneracy, JHEP 05 (2009) 121 [arXiv:0810.3472] [SPIRES].

[70] S. Murthy and B. Pioline, A Farey tale for $N=4$ dyons, JHEP 09 (2009) 022 [arXiv: 0904.4253] [SPIRES].

[71] J.M. Maldacena and A. Strominger, $A d S_{3}$ black holes and a stringy exclusion principle, JHEP 12 (1998) 005 [hep-th/9804085] [SPIRES].

[72] D. Gaiotto, A. Strominger and X. Yin, From $A d S_{3} / C F T_{2}$ to black holes /topological strings, JHEP 09 (2007) 050 [hep-th/0602046] [SPIRES].

[73] B. Chandrasekhar, Black Hole Partition Function using Hybrid Formalism of Superstrings, arXiv: 0811.1758 [SPIRES].

[74] A. Dabholkar, S. Murthy and D. Zagier, Quantum black holes, wall crossing and mock modular forms, in preparation.

[75] V. Cortes, C. Mayer, T. Mohaupt and F. Saueressig, Special geometry of Euclidean supersymmetry. I: Vector multiplets, JHEP 03 (2004) 028 [hep-th/0312001] [SPIRES].

[76] H. Lu, C.N. Pope and J. Rahmfeld, A Construction of Killing spinors on $S^{n}$, J. Math. Phys. 40 (1999) 4518 [hep-th/9805151] [SPIRES]. 